\title{
La relación entre la auditoría, la corrupción política y la transparencia informativa: un análisis comparativo de las Comunidades Autónomas españolas
}

\author{
José Manuel Vela-Bargues ${ }^{\mathrm{a}, \mathrm{b}}$, Fernando Polo-Garrido ${ }^{\mathrm{a}, \mathrm{b}}$, Emilio J. de la Higuera ${ }^{\mathrm{c}}$, Gemma \\ Pérez-López ${ }^{d}$
}

a) Departamento de Economía y Ciencias Sociales, Facultad de Administración y Dirección de Empresas, Universitat Politècnica de València, Valencia, SPAIN. b) Centro de Investigación en Gestión de Empresas (CEGEA), Universitat Politècnica de València, Valencia, SPAIN.

c) Departamento de Economía Financiera y Contabilidad, Facultad de Ciencias Sociales y Jurídicas, Campus de Melilla, SPAIN.

d) Departamento de Economía Financiera y Contabilidad, Facultad de Ciencias Económicas y Empresariales, Universidad de Granada, Granada, SPAIN.

\author{
${ }^{d}$ Corresponding author. \\ E-mail address: gemmapl@ugr.es
}

\section{A R T I C L E I N F O}

Article history:

Received 18 November 2019

Accepted 9 June 2020

Available online 1 January 2022

\section{Códigos JEL:}

Códig
H70
H72

Palabras clave

Auditoría externa

OCEX

Transparencia

Corrupción

Comunidades Autónomas

\section{R E S U M E N}

En la actualidad, las administraciones públicas están experimentando una tendencia hacia un mayor control y supervisión como consecuencia de las demandas ciudadanas y la situación financiera de los últimos años. Concretamente, las Comunidades Autónomas españolas han sufrido en gran medida los efectos de la crisis económica del año 2008 y en ellas se ha detectado un gran número de procesos de corrupción política, lo que ha centrado la atención de los expertos en su estudio. Por ello, el objetivo principal del presente trabajo consiste en evaluar el efecto que el nivel de implantación de la auditoría externa y el grado de transparencia informativa tienen sobre la corrupción política en la Administración Pública Autonómica española. Para ello, se realiza en primer lugar, una revisión del panorama actual del nivel de implantación de la auditoría externa en las Comunidades Autónomas, de su transparencia informativa y del nivel de corrupción política a nivel regional. En segundo lugar, se lleva a cabo un estudio exploratorio consistente en un análisis bivariante y un modelo de regresión. Los resultados del análisis bivariante muestran que existe una relación significativa entre el nivel de implantación de auditoría externa y transparencia. No obstante, el modelo de regresión nos indica que ni transparencia ni auditoría influyen significativamente en la corrupción, salvo cuando analizamos índices parciales de auditoría y transparencia ya que la adaptación al PGCP 2010 resulta tener un efecto significativo respecto a los casos de corrupción.

(C)2022 ASEPUC. Published by EDITUM - Universidad de Murcia. This is an open access article under the CC BY-NC-ND license (http://creativecommons.org/licenses/by-nc-nd/4.0/).

The relationship between auditing, political corruption and information transparency: a comparative analysis of the Spanish Autonomous Communities A B S T R A C T

Nowadays, public administrations are experiencing a trend towards greater control and supervision as a result of citizen demands and the financial situation of recent years. Specifically, the Spanish Autonomous Communities have suffered greatly from the effects of the economic crisis of 2008 and they have detected a large number of political corruption processes, which has focused the attention of experts. Therefore, the main objective of this work is to evaluate the effect that the level of implementation of external auditing and the degree of information transparency have on political corruption in the Spanish Autonomous Public Administration. To do this, firstly, a review of the current level of implementation of external auditing in the Autonomous Communities, its transparency level and the degree of political corruption at the regional level is carried out. Secondly, an exploratory study consisting of a bivariate analysis and a regression model is performed. The results of the bivariate analysis show that there is a significant relationship between the level of external audit implementation and transparency. However, the regression model indicates that neither transparency nor auditing has a significant influence on corruption, except when we analyze partial audit and transparency indexes since the 2010 PGCP turns out to have a significant effect regarding corruption cases.

(C)2022 ASEPUC. Publicado por EDITUM - Universidad de Murcia. Este es un artículo Open Access bajo la licencia CC BY-NC-ND (http://creativecommons.org/licenses/by-nc-nd/4.0/). 


\section{Introducción}

La creciente demanda por parte de los ciudadanos de un mayor nivel de información (Chaikin \& Sharman, 2009; Guillamón et al., 2011; Ríos et al., 2017) ha provocado que en los últimos años hayan proliferado los estudios centrados en el análisis de la transparencia de las Administraciones Públicas (Pilcher et al., 2008; Curry et al., 2014). Así, han surgido trabajos como el de Da Cruz \& Marques (2014) o GarridoRodríguez et al. (2017; 2019) que proponen nuevos métodos para medir la transparencia informativa en las administraciones públicas locales; mientras que, también pueden hallarse en la literatura otros estudios que buscan dar una respuesta al efecto que tiene la transparencia sobre ciertos aspectos claves de las administraciones públicas, como el estudio de Grimmelikhuijsen et al. (2013), centrado en la confianza en el Gobierno. Además, el análisis de la transparencia se ha llevado a cabo atendiendo a distintos niveles de la Administración Pública, así como a servicios específicos, como puede observarse en los trabajos de De Fine-Licht (2011), Royo et al. (2019) o Benito et al. (2021). En concreto, la literatura dedicada al análisis de la transparencia a nivel de las Comunidades Autónomas en España es bastante escasa, destacando los trabajos de Bellod (2008) y Ríos et al. (2019), que se centran en el ámbito presupuestario.

A su vez, se han ido desarrollando en los distintos países normativas propias que regulan la forma de cumplir con los requisitos de la información publicada y de libre acceso para los ciudadanos, no existiendo una regulación a nivel global de transparencia, concurriendo, incluso, una gran diversidad a nivel regional y local dentro de un mismo país, en cuanto a normativa que regule esta cuestión. Pese a ello, la transparencia de las entidades públicas ha experimentado una gran evolución (Da Cruz et al., 2016), hecho que ha demostrado ser fundamental en la rendición de cuentas (Shim \& Eom, 2008; Cullier \& Piotrowski, 2009). Con esta nueva situación, en la que el acceso a la información contable de carácter económico, social y político es más abierto, el control externo de las Administraciones Públicas puede haber experimentado cambios como consecuencia de la misma. No obstante, no existen estudios previos que hayan analizado la relación entre el nivel de transparencia y la auditoría externa de las entidades públicas.

Por otro lado, la transparencia puede relacionarse con el nivel de corrupción política en las administraciones públicas. La corrupción política comprende generalmente actividades ilegales, que salen a la luz a través de escándalos, investigaciones o enjuiciamientos (Benito et al., 2018). En este sentido, autores como Kolstad \& Wiig (2009) han demostrado que la transparencia es un aspecto clave en la lucha contra la corrupción, evitando que los fondos públicos sean empleados de forma inadecuada al no aplicarse por el bien común (Magdaleno y García-García, 2014). En concreto, estudios como los de Lindstedt \& Naurin (2010) y Montes \& Luna (2020) demuestran que la transparencia reduce el nivel de corrupción política.

Por su parte, la auditoría externa también juega un importante papel en la lucha contra la corrupción (Kaufmann, 2000), atrayendo cada vez más un elevado interés social, a través de sus informes de fiscalización, por considerarse estos de gran utilidad para evaluar los casos de corrupción política y administrativa que se han erigido en los últimos años (Chaikin \& Sharman, 2009; Guillamón et al., 2011). En este sentido, el análisis del impacto que la auditoría en el sector público tiene en la corrupción política son escasos (Gustavson \& Sundström, 2018). Los estudios de Duflo et al. (2012),
Duflo et al. (2013), Avis et al. (2018) y Gustavson \& Sundström (2018) obtienen que la auditoría puede reducir el nivel de corrupción por parte de los agentes públicos. Por este motivo, es necesario conocer el grado en que la auditoría externa se ha implantado en las Administraciones Públicas regionales, para conseguir así determinar cuál es el alcance de los informes de auditoría externa en este nivel de la Administración Pública, y si dichos informes ejercen influencia en los casos de corrupción política acaecidos en los últimos años dentro de las Comunidades Autónomas.

En vista de lo anterior, es evidente que la transparencia se encuentra estrechamente vinculada al ámbito de la rendición de cuentas (Meijer, 2003; Andersen, 2009; Villoria \& Cruz-Rubio, 2016), existiendo un triángulo en la gestión pública que "aglutina la transparencia, la contabilidad pública y el control externo" (Viñas, 2018), surgiendo así una nueva necesidad de investigación que permita demostrar la existencia o no de una relación entre el nivel de transparencia de las entidades públicas, el grado de implantación del control externo sobre las mismas y los casos de corrupción detectados en ellas, aspecto que no ha sido tratado con anterioridad en la literatura previa. Así, el objetivo fundamental de este trabajo es evaluar el efecto que el nivel de implantación de la auditoría externa y el grado de transparencia informativa tienen sobre la corrupción política, considerando para ello la situación de cada una de las Comunidades Autónomas españolas en estos tres ámbitos de estudio.

En este contexto, el trabajo se estructura en tres partes fundamentales. La primera de ellas se dedica a presentar un análisis comparativo del grado de implantación de la auditoría externa en las Comunidades Autónomas españolas (Vela \& Zafra-Gómez, 2019), así como de los niveles de transparencia informativa que existe a nivel regional y, por último, del número de casos de corrupción que se han detectado en cada una de ellas (Della Porta, 2017; Johnston, 2017; Benito et al., 2018). En este sentido, cabe destacar que el nivel de implantación de la auditoría en las Comunidades Autónomas es tremendamente dispar, caracterizándose su situación actual por la gran heterogeneidad existente en el alcance, contenido, estructura y fechas de presentación de los informes. Esta heterogeneidad convierte la iniciativa de llevar a cabo un análisis comparativo de este nivel de implantación a nivel regional en una tarea ciertamente compleja, pero de gran utilidad para establecer la relación existente entre transparencia, corrupción y control externo. Asimismo, es importante matizar que la situación de la corrupción política es también muy desigual, mientras que, las diferencias en los índices de transparencia son mucho menos dispares.

La segunda parte del trabajo presenta la parte empírica del mismo. En primer lugar, se ha realizado un estudio exploratorio de las posibles relaciones bivariantes entre auditoria, transparencia y corrupción. Seguidamente, se presenta el modelo de regresión que analiza el posible efecto de la auditoría y transparencia sobre la corrupción en las Comunidades Autónomas. Finalmente, la tercera y última parte comprende las principales conclusiones obtenidas.

\section{Auditoría externa, transparencia informativa y corrup- ción en las Comunidades Autónomas españolas}

Como ya se ha señalado en la sección de introducción del presente trabajo, la situación de las distintas Comunidades Autónomas es muy heterogénea, por lo que, previo análisis de la relación entre la auditoría externa, la transparencia y la corrupción, resulta necesario realizar un análisis comparativo de la situación de cada una de ellas. 
2.1. Análisis del nivel de implantación de la auditoría externa en las Comunidades Autónomas españolas

En la actualidad, aunque con un alcance diferente, todas las Comunidades Autónomas españolas llevan a cabo una auditoría externa de su Cuenta General, extendiendo en algunos casos su fiscalización al ámbito de la Administración Local y de otras entidades, como consorcios u otros entes fondos carentes de personalidad jurídica, agencias, fondo de compensación interterritorial o Universidades-. De las 17 Comunidades Autónomas, todas ellas excepto cinco disponen de un Órgano de Control Externo (OCEX). Las cinco Comunidades en las que la fiscalización es realizada por el Tribunal de Cuentas son: Cantabria, Castilla-La Mancha, Extremadura, Murcia y La Rioja, además de las ciudades autónomas de Ceuta y Melilla, que, a pesar de formar parte del Consejo de Política Fiscal y Financiera, no se han incluido en el presente trabajo dada esta condición. Hay que resaltar que la Comunidad de Castilla la Mancha suprimió su OCEX existente por Ley de CLM 1/2014 (Pérez-López, 2019a).

De acuerdo con el enfoque adoptado por Vela \& ZafraGómez (2019) en su trabajo "La investigación sobre la auditoría en el sector público en España", en el que se analizan los informes de fiscalización de las distintas Comunidades Autónomas relativos al ejercicio 2014 y publicados entre 2015 y 2018 , se han seleccionado seis variables fundamentales con el fin de poder establecer un indicador del grado de implantación de la auditoría externa (GIA) en cada una de las respectivas comunidades. A continuación, se describen cada una de estas variables, mostrando la Tabla 1 un resumen de la información de estas variables para cada comunidad. En este sentido, se mantienen las puntuaciones establecidas a cada variable siguiendo el informe de Vela \& Zafra-Gómez (2019), quienes, en función de la importancia relativa que cada una tiene sobre el grado de implantación de la auditoría externa, otorgan puntuaciones máximas de 0,5, 1 y 2 .

\section{- GIA1: Antigüedad del órgano de control externo.}

Esta variable mide la antigüedad del OCEX de las distintas Comunidades Autónomas, habiéndose otorgado una puntuación máxima de 0,5 (Vela \& Zafra-Gómez, 2019). En este sentido se considera que una mayor antigüedad del órgano de control externo se traduce en una mayor experiencia y tradición en la realización de trabajos de auditoría, especialmente en lo que se refiere a su número y alcance. Así, a las comunidades con un OCEX anterior a 1990 se les ha asignado una puntuación de 0,5 puntos y al resto 0 puntos.

- GIA2: Aplicación por parte de la Comunidad Autónoma de un Plan Contable adaptado al Plan General de Contabilidad Pública de 2010 (PGCP-2010).

Esta variable identifica a aquellas Comunidades Autónomas que han adaptado su normativa contable al PGCP-2010. En relación con esta variable, hay que señalar que en el ejercicio 2014, el cual se ha tomado como referencia, tan sólo dos Comunidades habían adaptado su normativa contable al PGCP-2010: El País Vasco y la Rioja (Vela y Zafra-Gómez, 2019). Andalucía lo hizo con posterioridad (Zafra-Gómez, 2019), y no ha sido hasta 2017 en que otras Comunidades, como la valenciana, declararon derogada su normativa contable para poder empezar a aplicar el PGCP-2010, aunque más que por iniciativa propia, por la exigencia del programa para la aplicación del compartimento Fondo de Liquidez Autonómico del Modelo de Financiación de las Comunidades Autónomas para el ejercicio 2017 (Vela et al., 2018). En 2014, la aplicación por parte de las Comunidades Autónomas de Planes contables diferentes provocó que la información recogida en sus cuentas generales no resultara en algunos casos comparable.

La puntuación máxima que se ha asignado a esta variable es de 2 puntos -otorgándosele a las Comunidades del País Vasco y La Rioja (Tabla 1)- al considerarse que la adaptación de la normativa contable en cada Comunidad Autónoma al PGCP2010 supone un avance, pues la información contable resulta más comparable con la del resto de Administraciones (estatal y local) y se adaptada a las normas internacionales de Contabilidad Pública (Vela \& Zafra-Gómez, 2019).

\section{- GIA3: Plazo de Rendición de la Cuenta General.}

Esta variable mide el nivel de cumplimiento de los plazos de presentación de la Cuenta General por parte de las distintas Comunidades Autónomas. Cabe destacar que, al igual que sucedía con la normativa contable aplicable en las diferentes Comunidades Autónomas españolas en el ejercicio 2014, los plazos de rendición de sus Cuentas Generales tampoco son uniformes.

Esta variable comprende un máximo de 1 punto, para cuya asignación se ha tenido en cuenta el requisito de la información contable relativo a la oportunidad de la información (Vela \& Zafra-Gómez, 2019), otorgándole a aquellas comunidades en las que no se ha registrado ninguna incidencia con respecto al plazo de presentación de la Cuenta General el máximo valor de esta variable (1 punto); mientras que, aquellas en las que se han producido incidencias obtienen 0,5 puntos, y las comunidades que hayan incumplido el plazo tendrán asignado un valor de 0 puntos.

- GIA4: Fecha de aprobación del Informe de Auditoría por el correspondiente órgano de control externo.

Esta variable mide los plazos en los que los diferentes OCEX aprueban su informe de la Cuenta General de cada Comunidad Autónoma, los cuales son muy dispares (véase Tabla 1). Esta situación puede afectar a la oportunidad de los informes de fiscalización e igualmente a la posterior comparabilidad de los datos contenidos en los mismos, es por ello que en aquellos casos en los que la fecha de aprobación de los respectivos informes de auditoría se aleja más de la fecha de cierre del ejercicio auditado, su oportunidad resulta menor.

Teniendo en cuenta la anterior circunstancia, a las Comunidades en las que dicho plazo ha sido inferior a 12 meses se les ha otorgado el valor máximo de 1 punto; mientras que, cuando el plazo ha estado comprendido entre 12 y 24 meses, se le asigna 0,5 puntos, y 0 puntos si el plazo supera los dos años (Vela \& Zafra-Gómez, 2019).

- GIA5: Alcance de la auditoría en el informe correspondiente a la Administración General (consejerías y organismos autónomos administrativos).

Esta variable trata de hacer una valoración sobre el nivel de alcance de la auditoría llevada a cabo en el informe correspondiente a la Administración General; sin embargo, cabe señalar que, pese a que todas las Comunidades Autónomas llevan a cabo auditorías de legalidad o cumplimiento y un análisis del proceso de rendición de cuentas, éstas son auditorías centradas fundamentalmente en la ejecución del presupuesto y otros aspectos puntuales como el área de personal, la contratación o la gestión de la deuda y avales. La realización de auditorías de regularidad en la que se expresa 
una opinión con referencia a la imagen fiel y las correspondientes salvedades en su caso, no se encuentra generalizada todavía.

En el estudio de Vela \& Zafra-Gómez (2019), se puede observar que algunas Comunidades Autónomas expresan una opinión referida exclusivamente al cumplimiento de la legalidad, tratándose por tanto de informes vinculados al objetivo de rendición de cuentas o auditorías de cumplimiento. Sin embargo, en otras, además de la legalidad, se lleva a cabo una auditoría de regularidad en la que la opinión también se refiere al ámbito de la imagen fiel en el marco de las cuentas anuales que integran su Cuenta General, con expresa alusión a este concepto o con un alcance asimilable. Por lo tanto, se han asignado a las Comunidades que llevan a cabo de forma adicional esta auditoría de regularidad, 2 puntos, mientras que al resto se les ha asignado 1 punto.

- GIA6: Auditoría en el ámbito de la Administración Local.

Con esta variable se pretende valorar el nivel de auditoría al que cada una de las Comunidades Autónomas somete a las entidades locales que la conforman. Hay que destacar que la totalidad de órganos de control externo presentan informes de fiscalización de corporaciones locales relativos al ejercicio 2014, aunque en realidad no se trata de trabajos de auditoría, sino más bien de control de la rendición de cuentas y definición de algunos indicadores a partir de la información contenida en sus cuentas anuales (Vela \& Zafra-Gómez, 2019). Obviamente, Auditoría y Rendición de Cuentas no son procesos equivalentes (Vela et al., 2016). En España, la Auditoría externa de las Cuentas de las Administraciones Locales no es obligatoria, siendo competencia exclusiva del Tribunal de Cuentas. Ante la falta de medios existente, una gran mayoría de las corporaciones locales españolas no son auditadas de forma regular (Dasí et al., 2018), salvo que, puntualmente, alguno de los órganos de control externo decida incluir algún trabajo en este ámbito dentro de sus planes anuales de actuación.

Con respecto al ejercicio 2014, la realización de trabajos de auditoría de regularidad en el ámbito de la Administración Local, con mención expresa sobre la imagen fiel, no resultó ser una práctica generalizada (Vela \& Zafra-Gómez, 2019), aunque debe destacarse la intensa actividad llevada a cabo por los dos órganos de control externo de las Comunidades Forales de Navarra y País Vasco, así como por Aragón y la Comunidad Autónoma de Islas Baleares con respecto a sus Consejos Insulares. Otras Comunidades como Andalucía, Asturias, Canarias, Cataluña, Comunidad Valenciana, Murcia cuentan con controles parciales que afectan a algunos de sus Ayuntamientos, mientras en el caso de Cantabria, Castilla-La Mancha, Castilla y León, Extremadura, Galicia y Madrid, la actividad de control es prácticamente nula en este ámbito.

El valor máximo para esta variable es de 1,5 puntos de manera que aquellas comunidades que llevan a cabo la auditoría de la administración local reciben la máxima puntuación; mientras que, las que la realizan con un alcance parcial obtienen 1 punto, a aquellas comunidades que realizan esta auditoría de forma testimonial se les asigna 0,5 puntos y 0 puntos, para las que no realizan este control (Vela \& ZafraGómez, 2019).

Una vez explicadas cada una de las variables y teniendo en cuenta la información presentada en la Tabla 1 , se presenta la Tabla 2 que recoge las puntuaciones obtenidas por cada Comunidad Autónoma para cada una de las variables establecidas, siguiendo la metodología adoptada en Vela \& Zafra-Gómez (2019), así como sus puntuaciones globales, reflejando sobre un total de 8 puntos el nivel de implantación de la auditoría de cada Comunidad Autónoma, que adicionalmente se ha convertido sobre base 100 para facilitar su lectura. Como puede observarse, las comunidades que presentan un grado de implantación y alcance de la auditoría externa más satisfactorio son las de País Vasco, Navarra, Comunidad Valenciana, Canarias y Cataluña.

Del análisis de las puntuaciones obtenidas por cada Comunidad Autónoma, se desprenden unos resultados muy similares a los obtenidos en el trabajo de Vela \& Zafra-Gómez (2019), teniendo en cuenta que, en el mismo, se considera como variable adicional la transparencia, expresada en función del volumen de gasto público que es objeto de auditoría de regularidad (imagen fiel) en cada Comunidad Autónoma. En vista del objetivo y alcance del presente trabajo, se ha procedido a su exclusión, dado que el concepto de transparencia que se evalúa de forma separada en nuestro caso, resulta mucho más amplio, ya que como se expondrá a continuación, en este trabajo se ha obtenido de acuerdo con la metodología utilizada por "Transparency International España"(2016a).

\subsection{La Transparencia en las Comunidades Autónomas espa- ñolas}

En los últimos años, las administraciones públicas especialmente las comunidades autónomas- han sufrido diversos problemas como las dificultades para afrontar puntualmente sus pagos o el incremento de los niveles de presión fiscal (López-Hernández et al., 2018), los cuales han sido consecuencia de la crisis económica y financiera que comenzó en el año 2008. Dicha circunstancia es uno de los principales catalizadores que impulsaron una elevada demanda social de información y transparencia en la gestión pública por parte de la ciudadanía (Da Cruz \& Marques, 2014; Da Cruz et al., 2016). Así, las administraciones públicas han tenido que introducir herramientas como la transparencia, que en la actualidad se han convertido en un pilar esencial del proceso de rendición de cuentas en el ámbito público (Ormaetxea, 2014).

Por otro lado, el marco normativo que regula los mecanismos de transparencia en las administraciones públicas es diferente en cada país de la Unión Europea. Concretamente, en el contexto español, la transparencia está regulada por la Ley 19/2014, de 29 de diciembre, "de transparencia, acceso a la información pública y buen gobierno", la cual supuso un claro intento de dar respuesta a dichas demandas, facilitando la creación de un organismo con personalidad jurídica propia, que se constituyó bajo la denominación de Consejo de Transparencia y Buen Gobierno, cuyas funciones aparecen reguladas en el art. 3 del Real Decreto 919/2014, de 31 de octubre. Asimismo, también han sido promulgadas Leyes de Transparencia a nivel autonómico, aunque a efectos del objeto del presente trabajo, las mismas no ofrecen diferencias significativas con respecto a la legislación estatal.

Respecto al concepto de transparencia, muchas han sido las definiciones que se han adoptado en la literatura previa acerca del mismo (Kaufmann \& Kraay, 2002; Matheson, 2002; Ortiz, 2012). Para el desarrollo del presente trabajo, partimos de la definición de transparencia de acuerdo con la Ley 19/2014, de 29 de diciembre, de transparencia, acceso a la información pública y buen gobierno, que la define como "la acción proactiva de la Administración de dar a conocer la información relativa a sus ámbitos de actuación y sus obligaciones, con carácter permanente y actualizado, de la forma que resulte más comprensible para las personas y mediante los instrumentos de difusión que les permitan un amplio y fácil acceso a los datos y faciliten su participación en los asuntos públicos." 
Tabla 1. Grado de implantación auditoría. Variables consideradas.

Cuadro Comparativo ejercicio. 2014

\begin{tabular}{|c|c|c|c|c|c|c|}
\hline & $\begin{array}{l}\text { Órgano Control Externo } \\
\text { (OCEX) } \\
\text { Año de creación }\end{array}$ & $\begin{array}{l}\text { Normativa contable } \\
\text { aplicable A.G. }\end{array}$ & $\begin{array}{l}\text { Rendición de la } \\
\text { cuenta general } \\
\text { en plazo }\end{array}$ & $\begin{array}{l}\text { Fecha de aprobación } \\
\text { informe Cuenta } \\
\text { General por OCEX }\end{array}$ & $\begin{array}{c}\text { Auditoría } \\
\text { Administración } \\
\text { General }\end{array}$ & $\begin{array}{l}\text { Auditorías del OCEX sobre } \\
\text { entidades locales } \\
\text { LEGALIDAD/REGULARIDAD }\end{array}$ \\
\hline ANDALUCIA & $\begin{array}{c}\text { Cámara de Cuentas } \\
\text { Andalucía } \\
1988\end{array}$ & $\begin{array}{c}2015 \\
\text { PGCP Andalucía } \\
\text { Adaptado al PGCP } 2010\end{array}$ & SI & $\begin{array}{l}23 \text { de febrero de } \\
2016\end{array}$ & SI LEGALIDAD & $\begin{array}{c}\text { NO } \\
\text { Sólo auditorías parciales con } \\
\text { alcance limitado }\end{array}$ \\
\hline ARAGÓN & $\begin{array}{c}\text { Cámara de Cuentas de } \\
\text { Aragón } \\
2009\end{array}$ & $\begin{array}{c}1994 \\
\text { PGCP DE Aragón. } \\
\text { NO ADAPTADO AL PGCP }\end{array}$ & NO & $\begin{array}{l}24 \text { de junio de } \\
2016\end{array}$ & $\begin{array}{l}\text { SI LEGALIDAD } \\
\text { SI REGULARIDAD }\end{array}$ & $\begin{array}{c}\text { SI } \\
\text { LEGALIDAD Y REGULARIDAD }\end{array}$ \\
\hline ASTURIAS & $\begin{array}{c}\text { Sindicatura de Ctas. del P. de } \\
\text { Asturias } \\
2003\end{array}$ & $\begin{array}{l}2004 \text { PGCP Asturias. } \\
\text { NO ADAPTADO AL PGCP } \\
2010\end{array}$ & SI & $\begin{array}{c}3 \text { de febrero de } \\
2016\end{array}$ & $\begin{array}{l}\text { SI LEGALIDAD } \\
\text { SI REGULARIDAD }\end{array}$ & $\begin{array}{c}\text { NO } \\
\text { Sólo auditorías parciales con } \\
\text { alcance limitado }\end{array}$ \\
\hline ISLAS BALEARES & $\begin{array}{c}\text { Sindicatura de Cuentas de } \\
\text { las Islas Baleares } \\
2004\end{array}$ & $\begin{array}{c}\text { PGCP } 1999 \text { NO } \\
\text { ADAPTADO AL PGCP } \\
2010\end{array}$ & SI & $\begin{array}{l}8 \text { de septiembre de } \\
2016\end{array}$ & $\begin{array}{l}\text { SI LEGALIDAD } \\
\text { SI REGULARIDAD } \\
\text { IMAGEN FIEL }\end{array}$ & $\begin{array}{c}\text { REGULARIDAD } \\
\text { Si en el Caso de Consejos } \\
\text { Insulares } \\
\text { No se auditan Ayuntamientos }\end{array}$ \\
\hline ISLAS CANARIAS & $\begin{array}{c}\text { Audiencia de Cuentas de } \\
\text { Canarias. } \\
1989\end{array}$ & $\begin{array}{c}\text { PGCP } 1994 \text { NO } \\
\text { ADAPTADO AL PGCP } \\
2010\end{array}$ & SI & $\begin{array}{l}18 \text { de diciembre de } \\
2015\end{array}$ & SI LEGALIDAD & $\begin{array}{l}\text { NO } \\
\text { Sólo auditorías parciales con } \\
\text { alcance limitado }\end{array}$ \\
\hline CANTABRIA & $\begin{array}{l}\text { No existe. } \\
\text { Actúa el Tribunal de Cuentas }\end{array}$ & $\begin{array}{c}2000 \text { PGCP. } \\
\text { NO ADAPTADO AL PGCP } \\
2010\end{array}$ & SI & $\begin{array}{l}23 \text { de enero de } \\
2017\end{array}$ & SI LEGALIDAD & NO \\
\hline $\begin{array}{l}\text { CASTILLA-LA } \\
\text { MANCHA }\end{array}$ & $\begin{array}{c}\text { No existe. } \\
\text { Actúa el Tribunal de Cuentas } \\
\text { (1) }\end{array}$ & $\begin{array}{c}2000 \text { PGCP Cantabria. } \\
\text { NO ADAPTADO AL PGCP } \\
2010\end{array}$ & SI & $\begin{array}{l}24 \text { de enero de } \\
2017\end{array}$ & SI LEGALIDAD & NO \\
\hline $\begin{array}{l}\text { CASTILLA Y } \\
\text { LEÓN }\end{array}$ & $\begin{array}{l}\text { Consejo de Cuentas de } \\
\text { Castilla y León } \\
2002\end{array}$ & $\begin{array}{c}1996 \text { PGC de Castilla } \\
\text { León } \\
\text { NO ADAPTADO AL PGCP } \\
2010\end{array}$ & $\begin{array}{c}\text { SI } \\
\text { Con incidencias }\end{array}$ & $\begin{array}{l}24 \text { de noviembre de } \\
\text { 2016- }\end{array}$ & SI LEGALIDAD & NO \\
\hline CATALUÑA & $\begin{array}{c}\text { Sindicatura de Cuentas de } \\
\text { Cataluña } \\
1984\end{array}$ & $\begin{array}{l}1996 \text { PGCP Generalidad } \\
\text { de Cataluña } \\
\text { NO ADAPTADO AL PGCP } \\
2010\end{array}$ & $\begin{array}{c}\text { SI } \\
\text { Con incidencias }\end{array}$ & $\begin{array}{l}16 \text { de noviembre de } \\
2016\end{array}$ & $\begin{array}{l}\text { SI LEGALIDAD } \\
\text { SI REGULARIDAD } \\
\text { IMAGEN FIEL }\end{array}$ & $\begin{array}{c}\text { NO } \\
\text { Sólo auditorías parciales con } \\
\text { alcance limitado }\end{array}$ \\
\hline $\begin{array}{l}\text { COMUNIDAD } \\
\text { VALENCIANA }\end{array}$ & $\begin{array}{l}\text { Sindicatura de Cuentas Com. } \\
\text { Valenciana } \\
1985\end{array}$ & $\begin{array}{l}2001 \text { PGCP Generalitat } \\
\text { Valenciana } \\
\text { NO ADAPTADO AL PCCP } \\
2010\end{array}$ & SI & $\begin{array}{l}16 \text { de diciembre de } \\
2015\end{array}$ & $\begin{array}{l}\text { SI LEGALIDAD } \\
\text { SI REGULARIDAD } \\
\text { IMAGEN FIEL }\end{array}$ & $\begin{array}{l}\text { NO } \\
\text { Sólo auditorías parciales con } \\
\text { alcance limitado }\end{array}$ \\
\hline EXTREMADURA & $\begin{array}{l}\text { No existe. } \\
\text { Actúa el Tribunal de Cuentas }\end{array}$ & $\begin{array}{c}1999 \text { PGCP } \\
\text { EXTREMADURA } \\
\text { NO ADAPTADO EL PGCP } \\
2010\end{array}$ & $\begin{array}{c}\text { SI } \\
\text { Con incidencias }\end{array}$ & $\begin{array}{l}20 \text { de febrero de } \\
2017\end{array}$ & SI LEGALIDAD & NO \\
\hline GALICIA & $\begin{array}{c}\text { Consejo de Cuentas de } \\
\text { Galicia } \\
1985\end{array}$ & $\begin{array}{c}2001 \text { PGCP Galicia } \\
\text { NO ADAPTADO PGCP } \\
2010\end{array}$ & $\begin{array}{c}\text { SI } \\
\text { Con incidencias }\end{array}$ & 13 de julio de 2016 & $\begin{array}{l}\text { SI LEGALIDAD } \\
\text { SI REGULARIDAD } \\
\text { IMAGEN FIEL }\end{array}$ & NO \\
\hline MADRID & $\begin{array}{c}\text { Cámara de Ctas. de la } \\
\text { Comunidad de Madrid } \\
1999\end{array}$ & $\begin{array}{c}1996 \text { PGCP } \\
\text { Comunidad de Madrid. } \\
\text { NO ADAPTADO AL } \\
\text { PGCP } 2010\end{array}$ & $\begin{array}{c}\text { SI } \\
\text { Con incidencias }\end{array}$ & $\begin{array}{l}29 \text { de diciembre de } \\
2015\end{array}$ & SI LEGALIDAD & NO \\
\hline MURCIA & $\begin{array}{l}\text { No existe. } \\
\text { Actúa el Tribunal de Cuentas }\end{array}$ & $\begin{array}{l}1994 \text { PGCP } \\
\text { Com. de Murcia } \\
\text { NO ADAPTADO AL } \\
\text { PGCP } 2010\end{array}$ & $\begin{array}{c}\text { SI } \\
\text { Con incidencias }\end{array}$ & $\begin{array}{l}26 \text { de enero de } \\
2017\end{array}$ & SI LEGALIDAD & $\begin{array}{l}\text { NO } \\
\text { Sólo auditorías parciales con } \\
\text { alcance limitado }\end{array}$ \\
\hline NAVARRA & $\begin{array}{c}\text { Cámara de Comptos de } \\
\text { Navarra. } \\
1984\end{array}$ & $\begin{array}{l}\text { Ley Foral 13/2007 } \\
\text { NO ADAPTADA AL } \\
\text { PGP2010 }\end{array}$ & SI & $\begin{array}{l}5 \text { de noviembre de } \\
2015\end{array}$ & $\begin{array}{l}\text { SI LEGALIDAD } \\
\text { SI REGULARIDAD } \\
\text { IMAGEN FIEL }\end{array}$ & $\begin{array}{c}\text { SI } \\
\text { LEGALIDAD Y REGULARIDAD }\end{array}$ \\
\hline PAIS VASCO & $\begin{array}{c}\text { Tribunal Vasco de Cuentas } \\
\text { Públicas } \\
1988\end{array}$ & $\begin{array}{l}2012 \\
\text { PGCP del País Vasco. } \\
\text { ADAPTADO AL PGCP } \\
2010\end{array}$ & SI & $\begin{array}{l}26 \text { de mayo de } \\
2016\end{array}$ & $\begin{array}{l}\text { SI LEGALIDAD } \\
\text { SI REGULARIDAD }\end{array}$ & $\begin{array}{c}\text { SI } \\
\text { LEGALIDAD Y REGULARIDAD }\end{array}$ \\
\hline LA RIOJA & $\begin{array}{l}\text { No existe. } \\
\text { Actúa el Tribunal de Cuentas }\end{array}$ & $\begin{array}{l}\text { Orden 18/2013. PGCP la } \\
\text { Rioja. } \\
\text { ADAPTADO AL PCGP } \\
2010\end{array}$ & SI & $\begin{array}{l}24 \text { de enero de } \\
2017\end{array}$ & SI LEGALIDAD & N/A \\
\hline
\end{tabular}

Fuente: Vela \& Zafra-Gómez (2019) y elaboración propia. 
Tabla 2. Grado de implantación auditoría. Valoración por Comunidades.

Cuadro Comparativo ejercicio 2014

\begin{tabular}{|c|c|c|c|c|c|c|c|c|}
\hline \multirow[b]{2}{*}{$\begin{array}{l}\text { COMUNIDAD } \\
\text { AUTÓNOMA }\end{array}$} & \multicolumn{8}{|c|}{$\begin{array}{l}\text { GRADO DE IMPLANTACIÓN Y ALCANCE } \\
\text { DE LA AUDITORIA EXTERNA }\end{array}$} \\
\hline & $\begin{array}{c}\text { GIA } \\
1\end{array}$ & $\begin{array}{c}\text { GIA } \\
2\end{array}$ & $\begin{array}{c}\text { GIA } \\
3\end{array}$ & $\begin{array}{c}\text { GIA } \\
4\end{array}$ & $\begin{array}{c}\text { GIA } \\
5\end{array}$ & $\begin{array}{c}\text { GIA } \\
6\end{array}$ & $\begin{array}{l}\text { TOTAL } \\
\text { GIA }\end{array}$ & $\begin{array}{c}\text { TOTAL } \\
\text { GIA } \\
\text { S/100 }\end{array}$ \\
\hline ANDALUCIA & 0,5 & 0 & 1 & 0,5 & 1 & 1 & 4 & 50 \\
\hline ARAGON & 0 & 0 & 0 & 0,5 & 2 & 1,5 & 4 & 50 \\
\hline ASTURIAS & 0 & 0 & 1 & 0,5 & 2 & 1 & 4,5 & 56,25 \\
\hline BALEARES & 0 & 0 & 1 & 0,5 & 2 & 1,5 & 5 & 62,50 \\
\hline CANARIAS & 0,5 & 0 & 1 & 1 & 1 & 1 & 4,5 & 56,25 \\
\hline CANT & 0 & 0 & 1 & 0 & 1 & 0 & 2 & 25 \\
\hline CASTILLA L & 0 & 0 & 1 & 0 & 1 & 0 & 2 & 25 \\
\hline CASTILLA LEÓN & 0 & 0 & 0,5 & 0,5 & 1 & 0 & 2 & 25 \\
\hline CATALUÑA & 0,5 & 0 & 0,5 & 0,5 & 2 & 1 & 4,5 & 56.25 \\
\hline $\begin{array}{l}\text { COMUNIDAD } \\
\text { VALENCIANA }\end{array}$ & 0,5 & 0 & 1 & 1 & 2 & 1 & 5,5 & 68,75 \\
\hline EXTREMADURA & 0 & 0 & 0,5 & 0 & 1 & 0 & 1,5 & 18,75 \\
\hline GALICIA & 0,5 & 0 & 0,5 & 0,5 & 2 & 0 & 3,5 & 43,75 \\
\hline MAD & 0 & 0 & 0,5 & 1 & 1 & 0,5 & 3 & 37,5 \\
\hline MURCIA & 0 & 0 & 0,5 & 0 & 1 & 1 & 2,5 & 31,25 \\
\hline NAVARRA & 0,5 & 0 & 1 & 1 & 2 & 1 & 5,5 & 68,75 \\
\hline & 0,5 & 2 & 1 & 0,5 & 1 & 1,5 & 6,5 & 81,25 \\
\hline LA RIOJA & 0 & 2 & 1 & 0 & 1 & 0 & 4 & 50 \\
\hline
\end{tabular}

Fuente: Vela \& Zafra-Gómez (2019)

GIA 1: Antigüedad del órgano de control externo

GIA 2: Aplicación del Plan General de Contabilidad adaptado a PGCP 2010

GIA 3: Plazo de rendición de la Cuenta General

GIA 4: Fecha de aprobación del Informe de Auditoría por el correspondiente órgano de control externo

GIA 5: Alcance de la Auditoría de la Administración General

GIA 6: Auditorías en el ámbito de la Administración Local

Por otro lado, se han elaborado diversas aproximaciones con el fin de medir la transparencia en el ámbito de las administraciones públicas, como el índice llamado DYNTRA (Dynamic Transparency Index), el Mapa Infoparticipa (Universitat Autónoma de Barcelona) o el Test de Aplicación Municipal de la Ley de Transparencia elaborado por la Universidad Rey Juan Carlos (Da Cruz \& Marques, 2016). No obstante, en términos de transparencia, la organización que con mayor profusión en el tiempo ha venido encargándose de la elaboración de índices de transparencia en el ámbito público es "Transparency International España", que establece cinco índices (Lizcano, 2016) referidos a Ayuntamientos (ITA), Gestión del Agua (INTRAG), Comunidades Autónomas (INCAU), Diputaciones (INDIP) y Parlamentos (IPAR). En la actualidad, se publican además índices específicos para Clubs de Fútbol, Federaciones Deportivas, Empresas Públicas y Partidos Políticos.

En el caso de las Comunidades Autónomas españolas, el índice de transparencia INCAU persigue evaluar la transparencia de los gobiernos de las diferentes Comunidades Autónomas e impulsar y propiciar el aumento de la información que estas instituciones ofrecen a los ciudadanos y la sociedad en su conjunto. A pesar de sus limitaciones (Magdaleno y García-García, 2014; Villoria, 2015), se trata del índice más utilizado, teniendo en cuenta que viene elaborándose de forma periódica desde 2010 .

Los Índices de Transparencia que elabora y publica "Transparencia Internacional España" están exclusivamente dirigidos a medir el nivel de transparencia de las correspondientes instituciones públicas, y ello a través de la evaluación de los datos y la información que hacen pública en su página web en relación con los ochenta indicadores y áreas de transpa- rencia de cada Índice. La metodología utilizada se basa en el envío de cuestionarios a cada Comunidad Autónoma y la asignación de puntuaciones a cada indicador (INCAU, 2016). Así, considerando el objetivo principal del presente trabajo, y la amplia y desglosada información disponible para el caso específico de las Comunidades Autónomas, se ha optado por utilizar los datos publicados para el ejercicio 2016, ya que es el último informe publicado hasta la fecha (INCAU, 2016). La fecha de publicación del informe coincide con el período de publicación de los Informes de Auditoría en los que se basa la medición del grado de implantación de la auditoría externa en las diferentes Comunidades Autónomas, salvo en los casos de aquellas que son auditadas por el Tribunal de Cuentas (Cantabria, Castilla-León, Extremadura, Murcia y la Rioja), que se publicaron en 2017. En cualquier caso, los informes publicados en 2016 no ofrecen diferencias sustanciales en cuanto a su estructura y alcance con respecto a los de 2017.

Las seis áreas de transparencia que se evalúan en el INCAU (2016) son las siguientes:

A) Transparencia activa e información sobre la Comunidad Autónoma.

B) Página web, relaciones con los ciudadanos y la sociedad, y participación ciudadana.

C) Transparencia económico-financiera.

D) Transparencia en las contrataciones, convenios, subvenciones y costes de los servicios.

E) Transparencia en materias de Ordenación del territorio, urbanismo y obras públicas.

F) Derecho de acceso a la información.

Es evidente que, para el objetivo del presente trabajo, las áreas más relevantes son las C, D y F, aunque la Tabla 3 resume la puntuación relativa a las seis áreas, así como la evaluación global obtenida por cada Comunidad Autónoma.

Como se desprende de la Tabla 3, las Comunidades Autónomas con mayor nivel de transparencia (con puntuación superior a 97 puntos) son las de País Vasco, La Rioja, Madrid, Cataluña y Baleares. De estas cinco comunidades, dos de ellas (Cataluña y País Vasco) aparecen también como las que presentan un grado de implantación de la auditoría externa más satisfactorio (Tabla 2), situación que no ocurre en el caso de las otras tres.

Por otra parte, entre las Comunidades con menor nivel de transparencia, destacan Navarra -que cuenta con un nivel de implantación de la auditoría externa muy satisfactorio (Tabla 2)-, Extremadura y Aragón -cuyo nivel de implantación de la auditoría externa resulta menos satisfactorio que en el caso navarro-

Si desde un punto doctrinal y conceptual, transparencia, control externo y rendición de cuentas constituyen conceptos claramente vinculados, los datos obtenidos corroboran esta relación en el caso de muy pocas comunidades, sin que a nivel global pueda establecerse una clara correlación. Ello ocurre incluso si consideramos el índice de transparencia parcial TPC referido a la información económico-financiera.

\subsection{Casos de corrupción política}

Como se ha mencionado anteriormente, la implantación de la auditoría externa y los índices de transparencia en las Comunidades Autónomas españolas han estado influenciadas por el entorno social y político. En este sentido, existen multitud de factores que pueden influir en la inclusión de estos mecanismos de control en las administraciones públicas, siendo la corrupción uno de los más ampliamente estudiados en la literatura previa (Tanzi, 1998; Treisman, 2007). 
Tabla 3. Índices de Transparencia por Comunidad Autónoma

\begin{tabular}{lccccccc}
\hline & \multicolumn{6}{c}{ AREAS DE TRANSPARENCIA } \\
\cline { 2 - 7 } $\begin{array}{l}\text { COMUNIDAD } \\
\text { AUTÓNOMA }\end{array}$ & TPA & TPB & TPC & TPD & TPE & TPF & $\begin{array}{c}\text { TP } \\
\text { GLOBAL }\end{array}$ \\
\hline ANDALUCIA & 94,7 & 95,8 & 93,8 & 100 & 95 & 100 & 96,3 \\
ARAGON & 84,2 & 91,7 & 87,5 & 84,6 & 100 & 90 & 88,8 \\
ASTURIAS & 92,1 & 100 & 96,9 & 92,3 & 100 & 80 & 93,8 \\
BALEARES & 100 & 100 & 100 & 88,5 & 100 & 100 & 98,1 \\
CANARIAS & 100 & 100 & 96,9 & 80,8 & 90 & 100 & 95 \\
CANTABRIA & 100 & 83,3 & 100 & 84,6 & 100 & 100 & 95 \\
CASTILLA LM & 89,5 & 100 & 90,6 & 84,6 & 100 & 100 & 90,6 \\
CASTILLA LEÓN & 94,7 & 100 & 90,6 & 88,5 & 95 & 100 & 94,4 \\
CATALUNA & 100 & 100 & 96,9 & 92,3 & 100 & 100 & 98,1 \\
COMUNIDAD & 100 & 100 & 84,4 & 84,6 & 100 & 100 & 94,4 \\
VALENCIANA & & & & & & & \\
EXTREMADURA & 89,5 & 91,7 & 87,5 & 84,6 & 85 & 90 & 88,1 \\
GALICIA & 92,1 & 87,5 & 100 & 84,6 & 85 & 100 & 91,9 \\
MADRID & 94,7 & 100 & 100 & 100 & 95 & 100 & 98,1 \\
MURCIA & 100 & 95,8 & 100 & 92,3 & 95 & 95 & 96,9 \\
NAVARRA & 86,8 & 95,8 & 75 & 80,8 & 80 & 70 & 81,9 \\
PAIS VASCO & 100 & 100 & 100 & 100 & 100 & 100 & 100 \\
LA RIOJA & 94,7 & 100 & 100 & 100 & 95 & 95 & 97,5 \\
\hline
\end{tabular}

Fuente: Transparencia Internacional España (2016b).

TPA: Transparencia activa e información sobre la Comunidad Autónoma

TPB: Página Web, relaciones con los ciudadanos y la sociedad, y participación ciudadana

TPC: Transparencia Económico-Financiera

TPD: Transparencia en Convenios, Subvenciones y Costes de los Servicios.

TPE: Transparencia en materias de ordenación del territorio, urbanismo y obras púb: Tran

TPF: Derecho de acceso a la información.

Así desde un punto de vista teórico, los conceptos de transparencia y corrupción se muestran estrechamente vinculados, especialmente considerando el papel preventivo que la transparencia y la rendición de cuentas pueden ejercer sobre el nivel de corrupción (Cabezas, 2010; Cuadrado-Ballesteros \& Peña-Miguel, 2020) y su trascendencia informativa (Reylea, 2009).

En relación con el concepto de corrupción, aunque no existe un acuerdo unánime en la doctrina, una de las más utilizadas es la desarrollada por Transparency International (2018), que lo define como "el abuso de poder encomendado para beneficio privado". Del mismo modo, las definiciones realizadas por la literatura previa (Johnston, 1996), están orientadas generalmente a la existencia de un abuso de poder, malversación o búsqueda de un beneficio propio (Campos \& Pradhan, 2007; Golden \& Picci, 2005; Heidenheimer et al., 1989; Lambsdorff, 2005; Lindstedt \& Naurin, 2010; Van Klaveren, 1989).

En relación con el objetivo de este trabajo, se ha tratado de identificar los casos de corrupción en cada una de las diferentes Comunidades Autónomas, a partir de los datos publicados en el Repositorio del Consejo General del Poder Judicial, los cuales han sido sometidos a un proceso de depuración por Comunidad Autónoma. Este repositorio identifica los casos de corrupción a nivel local para cada Comunidad Autónoma, así como los casos de corrupción del gobierno regional. Además, enumera por trimestre los casos de corrupción con sentencia condenatoria (con o sin conformidad) dictada tanto por los Juzgados de lo Penal, las Audiencias Provinciales y los respectivos Tribunales Superiores de Justicia. Tal como se refleja en dicho repositorio, se consideran delitos de corrupción los siguientes: Ordenación del territorio, urbanismo y patrimonio histórico, Prevaricación de funcionarios públicos, Infidelidad en la custodia de documentos y violación de secretos, Cohecho, Tráfico de influencias, Malversación, Fraudes y exacciones ilegales, Negociaciones y actividades prohibidas a los funcionarios públicos y de los abusos en el ejercicio de
Tabla 4. Casos de Corrupción. Repositorio Consejo General del Poder Judicial

\begin{tabular}{|c|c|c|c|c|c|c|c|c|}
\hline & $\begin{array}{c}\text { ÓRG. } \\
\text { JUDICIAL }\end{array}$ & $\begin{array}{c}3 \mathrm{~T} \\
2015\end{array}$ & $\begin{array}{c}4 \mathrm{~T} \\
2015\end{array}$ & 2016 & 2017 & $\begin{array}{c}1 \mathrm{~T} \\
2018\end{array}$ & $\begin{array}{c}2 \mathrm{~T} \\
2018\end{array}$ & TOTAL \\
\hline \multirow{4}{*}{ ANDALUCIA } & JUZ. PENAL & 3 & 2 & 12 & 16 & 0 & 3 & 36 \\
\hline & AUD. PROV. & 1 & 1 & 10 & 9 & 3 & 1 & 25 \\
\hline & TSJ & 0 & 0 & 0 & 0 & 0 & 0 & 0 \\
\hline & TOTAL & 4 & 3 & 22 & 25 & 3 & 4 & 61 \\
\hline \multirow{4}{*}{ ARAGON } & JUZ. PENAL & 0 & 0 & 0 & 1 & 0 & 0 & 1 \\
\hline & AUD. PROV. & 0 & 0 & 4 & 1 & 0 & 0 & 5 \\
\hline & TSJ & 0 & 0 & 0 & 0 & 0 & 0 & 0 \\
\hline & TOTAL & 0 & 0 & 4 & 2 & 0 & 0 & 6 \\
\hline \multirow{4}{*}{ ASTURIAS } & JUZ. PENAL & 0 & 0 & 0 & 0 & 1 & 1 & 2 \\
\hline & AUD.PROV. & 0 & 2 & 1 & 2 & 0 & 0 & 5 \\
\hline & TSJ & 0 & 0 & 0 & 0 & 0 & 0 & 0 \\
\hline & TOTAL & 0 & 2 & 1 & 2 & 1 & 1 & 7 \\
\hline \multirow{4}{*}{ BALEARES } & JUZ. PENAL & 0 & 0 & 4 & 5 & 1 & 1 & 11 \\
\hline & AUDS.PROV. & 0 & 2 & 7 & 12 & 0 & 0 & 21 \\
\hline & TSJ & 0 & 0 & 0 & 0 & 0 & 0 & 0 \\
\hline & TOTAL & 0 & 2 & 11 & 17 & 1 & 1 & 32 \\
\hline \multirow{4}{*}{ CANARIAS } & JUZ. PENAL & 0 & 0 & 5 & 0 & 1 & 1 & 7 \\
\hline & AUD. PROV. & 1 & 0 & 4 & 8 & 0 & 0 & 13 \\
\hline & TSJ & 0 & 0 & 0 & 1 & 0 & 0 & 1 \\
\hline & TOTAL & 1 & 0 & 9 & 9 & 1 & 1 & 21 \\
\hline \multirow{4}{*}{ CANTABRIA } & JUZ. PENAL & 0 & 0 & 1 & 2 & 0 & 0 & 3 \\
\hline & AUD. PROV. & 0 & 0 & 0 & 0 & 0 & 0 & 0 \\
\hline & TSJ & 0 & 0 & 0 & 0 & 0 & 0 & 0 \\
\hline & TOTAL & 0 & 0 & 1 & 2 & 0 & 0 & 3 \\
\hline \multirow{4}{*}{ CASTILLA LM } & JUZ. PENAL & 0 & 0 & 0 & 0 & 1 & 0 & 1 \\
\hline & AUD. PROV. & 0 & 1 & 2 & 0 & 0 & 0 & 3 \\
\hline & TSJ & 0 & 0 & 0 & 0 & 0 & 0 & 0 \\
\hline & TOTAL & 0 & 1 & 2 & 0 & 1 & 0 & 4 \\
\hline \multirow{4}{*}{ CASTILLA LEÓN } & JUZ. PENAL & 0 & 0 & 2 & 4 & 1 & 0 & 7 \\
\hline & AUD. PROV. & 0 & 0 & 4 & 2 & 0 & 1 & 7 \\
\hline & TSJ & 0 & 1 & 0 & 0 & 0 & 0 & 1 \\
\hline & TOTAL & 0 & 1 & 6 & 6 & 1 & 1 & 15 \\
\hline \multirow{4}{*}{ CATALUÑA } & JUZ. PENAL & 1 & 0 & 1 & 0 & 0 & 0 & 2 \\
\hline & AUD. PROV. & 0 & 0 & 2 & 3 & 2 & 0 & 7 \\
\hline & TSJ & 0 & 0 & 0 & 0 & 0 & 0 & 0 \\
\hline & TOTAL & 1 & 0 & 3 & 3 & 2 & 0 & 9 \\
\hline \multirow{4}{*}{$\begin{array}{l}\text { COMUNIDAD } \\
\text { VALENCIANA }\end{array}$} & JUZ. PENAL & 0 & 0 & 3 & 3 & 3 & 1 & 10 \\
\hline & AUD.PROV. & 1 & 0 & 3 & 4 & 3 & 0 & 11 \\
\hline & TSJ & 0 & 0 & 0 & 1 & 0 & 0 & 1 \\
\hline & TOTAL & 1 & 0 & 6 & 8 & 6 & 1 & 22 \\
\hline & JUZ. PENAL & 0 & 0 & 3 & 1 & 0 & 0 & 4 \\
\hline & AUD. PROV. & 0 & 0 & 0 & 2 & 0 & 0 & 2 \\
\hline EXTREMADURA & TSJ & 0 & 0 & 0 & 0 & 0 & 0 & 0 \\
\hline & TOTAL & 0 & 0 & 3 & 3 & 0 & 0 & 6 \\
\hline & JUZ. PENAL & 0 & 0 & 3 & 1 & 0 & 0 & 4 \\
\hline GALJCIA & AUD.PROV. & 0 & 0 & 0 & 4 & 0 & 0 & 4 \\
\hline GALICIA & TSJ & 0 & 0 & 0 & 0 & 0 & 0 & 0 \\
\hline & TOTAL & 0 & 0 & 3 & 5 & 0 & 0 & 8 \\
\hline & JUZ. PENAL & 0 & 1 & 2 & 2 & 0 & 0 & 4 \\
\hline MADRID & AUD. PROV. & 0 & 0 & 1 & 1 & 0 & 0 & 2 \\
\hline IVIADKUD & TSJ & 0 & 0 & 0 & 0 & 0 & 0 & 0 \\
\hline & TOTAL & 0 & 0 & 3 & 3 & 0 & 0 & 6 \\
\hline & JUZ. PENAL & 0 & 0 & 0 & 0 & 1 & 0 & 1 \\
\hline & AUDS PROV. & 0 & 0 & 0 & 1 & 0 & 0 & 1 \\
\hline MURCIA & TSJ & 0 & 0 & 0 & 0 & 0 & 0 & 0 \\
\hline & TOTAL & 0 & 0 & 0 & 1 & 1 & 0 & 2 \\
\hline & JUZ. PENAL & 0 & 0 & 0 & 0 & 0 & 0 & 0 \\
\hline NAVAPRA & AUDS PROV. & 0 & 0 & 0 & 0 & 0 & 0 & 0 \\
\hline NAVARRA & TSJ & 0 & 0 & 0 & 0 & 0 & 0 & 0 \\
\hline & TOTAL & 0 & 0 & 0 & 0 & 0 & 0 & 0 \\
\hline & JUZ. PENAL & 0 & 0 & 0 & 1 & 0 & 0 & 1 \\
\hline PAIS VASCO & AUD. PROV. & 0 & 0 & 0 & 1 & 0 & 0 & 1 \\
\hline PAIS VASCU & TSJ & 0 & 0 & 0 & 0 & 0 & 0 & 0 \\
\hline & TOTAL & 0 & 0 & 0 & 2 & 0 & 0 & 2 \\
\hline & JUZ. PENAL & 0 & 0 & 0 & 0 & 0 & 0 & 0 \\
\hline I A PIOIA & AUD.PROV. & 0 & 0 & 0 & 0 & 0 & 0 & 0 \\
\hline LA KIUJA & TSJ & 0 & 0 & 0 & 0 & 0 & 0 & 0 \\
\hline & TOTAL & 0 & 0 & 0 & 0 & 0 & 0 & 0 \\
\hline
\end{tabular}

Fuente: CGPJ (2018), y elaboración propia.

su función, y la Corrupción en las transacciones comerciales internacionales. Por tanto, teniendo en cuenta lo explicado sobre la obtención de los datos que se realiza a través del Repositorio del Consejo General del Poder Judicial, se ha to- 
mado la información de los casos de corrupción de las Administraciones Locales y del propio gobierno autonómico para el período que transcurre desde el tercer trimestre de 2015 y el segundo semestre de 2018 (ver Tabla 4).

Como puede apreciarse en la Tabla 4, las Comunidades Autónomas que presentan un mayor número de casos juzgados con sentencia condenatoria son Andalucía, Baleares, Comunidad Valenciana, Canarias y Castilla y León, resultando especialmente significativo el caso de Navarra y la Rioja donde no se registró ningún caso durante el período considerado. Ahora bien, a efectos comparativos, no debemos olvidar que la comparación entre Comunidades se ve afectada por cuestiones como el tamaño en término de población, cuantía del presupuesto, insularidad o la composición y organización en provincias (uniprovinciales vs pluriprovinciales).

Por esta razón, se ha ponderado el número de casos de corrupción con el número de habitantes (año 2014) de cada Comunidad Autónoma, con el fin de facilitar un análisis comparativo más riguroso y razonable, tal como se recoge la Tabla 5. Se ha optado por el criterio de población al ser más homogéneo, dado que el carácter uniprovincial o cuantía del presupuesto pueden ofrecer algunas leves distorsiones ya que, por ejemplo, la Comunidad de Madrid (uniprovincial)

cuenta con un presupuesto y población mayor que el de algunas Comunidades pluriprovinciales.

En consecuencia, el valor para el número de casos ponderado ( $v n c p$ ) se ha calculado, redondeando a un decimal, de la forma siguiente:

$$
v n c p=\frac{\text { Casos de corrupcion CGPJ } * 10.000 .000}{\text { Numero de habitantes }}
$$

Finalmente, en la Tabla 6 se presenta un cuadro resumen de los factores analizados en el presente trabajo, mostrando en primer lugar los datos relativos al grado de planificación y alcance de la auditoría, seguido de las áreas de transparencia analizadas y, por último, se presenta el valor de los procedimientos de corrupción ponderados.
Tabla 5. Número de caso de corrupción CGPJ ponderado por población

\begin{tabular}{lccc}
\hline $\begin{array}{l}\text { COMUNIDAD } \\
\text { AUTÓNOMA }\end{array}$ & POBLACION & $\begin{array}{c}\text { TOTAL NÚMERO } \\
\text { CASOS } \\
\text { CORRUPCION } \\
\text { CGPJ }\end{array}$ & $\begin{array}{c}\text { VALOR N } \\
\text { CASOS } \\
\text { CORRUPCIÓN } \\
\text { PONDERADO }\end{array}$ \\
\hline ANDALUCIA & 8.402 .305 & 61 & 72,6 \\
ARAGÓN & 1.325 .385 & 6 & 45,3 \\
ASTURIAS & 1.061 .756 & 7 & 65,9 \\
BALEARES & 1.103 .442 & 32 & 290 \\
CANARIAS & 2.104 .815 & 21 & 99,8 \\
CANTABRIA & 588.656 & 3 & 60 \\
CATILLA LA & 2.078 .611 & 4 & 19,2 \\
MANCHA & 2.494 .790 & 15 & 60,1 \\
CASTILLA LEÓN & 7.518 .903 & 9 & 12 \\
CATALUÑA & 22 & 44 \\
COMUNIDAD & 5.004 .844 & 22 & 54,6 \\
VALENCIANA & 1.099 .632 & 6 & 29,1 \\
EXTREMADURA & 1.69 & 9,3 \\
GALICIA & 2.748 .695 & 8 & 13,6 \\
COMUNIDAD & 6.454 .440 & 6 & 0 \\
DE MADRID & 1.466 .818 & 2 & 0,1 \\
MURCIA & 640.790 & 0 & \\
NAVARRA & 2.188 .985 & 2 & 0 \\
PAIS VASCO & 319.002 & 0 & \\
RIOJA & & & \\
\hline
\end{tabular}

Fuente: CGPJ (2018), y elaboración propia

Como puede observarse en la Tabla 6, las relaciones entre nivel de auditoría, transparencia y casos de corrupción únicamente pueden establecerse con claridad en el caso del País Vasco, que cuenta con un alto nivel de transparencia e implantación de la auditoría que a su vez se traduce en un escaso valor de casos de corrupción.

Por su parte, las Comunidades de Canarias y Comunidad Valenciana ofrecen elevados niveles de implantación de la auditoría y valores medios de transparencia que coexisten con niveles ponderados relativamente elevados de casos de corrupción; mientras que, Cataluña y Navarra combinan reducidos valores ponderados de corrupción con elevados niveles de implantación de auditoría y de transparencia.

Tabla 6. Nivel de Auditoría, Transparencia y Corrupción. Cuadro resumen comparativo

\begin{tabular}{|c|c|c|c|c|c|c|c|c|c|c|c|c|c|c|c|c|}
\hline \multirow[b]{2}{*}{$\begin{array}{c}\text { COOCAS } \\
\text { COMUNIDAD } \\
\text { AUTÓNOMA }\end{array}$} & \multicolumn{8}{|c|}{$\begin{array}{l}\text { GRADO DE PLANIFICACION Y } \\
\text { ALCANCE DE LA AUDITORIA }\end{array}$} & \multicolumn{7}{|c|}{ AREAS DE TRANSPARENCIA } & \multirow[b]{2}{*}{$\begin{array}{c}\text { VALOR DE } \\
\text { PROCEDIMIENTOS } \\
\text { CORRUPCION } \\
\text { PONDERADO }\end{array}$} \\
\hline & $\begin{array}{c}\text { GIA } \\
1\end{array}$ & $\begin{array}{c}\text { GIA } \\
2\end{array}$ & $\begin{array}{c}\text { GIA } \\
3\end{array}$ & $\begin{array}{c}\text { GIA } \\
4\end{array}$ & $\begin{array}{c}\text { GIA } \\
5\end{array}$ & $\begin{array}{c}\text { GIA } \\
6\end{array}$ & $\begin{array}{l}\text { TOTAL } \\
\text { GIA }\end{array}$ & $\begin{array}{c}\text { TOTAL } \\
\text { GPA } \\
\text { S/100 }\end{array}$ & TPA & TPB & TPC & TPD & TPE & $\mathrm{TPF}$ & $\begin{array}{c}\text { TP } \\
\text { GLOBAL }\end{array}$ & \\
\hline ANDALUCIA & 0,5 & 0 & 1 & 0,5 & 1 & 1 & 4 & 50 & 94,7 & 95,8 & 93,8 & 100 & 95 & 100 & 96,3 & 72,6 \\
\hline ARAGON & 0 & 0 & 0 & 0,5 & 2 & 1,5 & 4 & 50 & 84,2 & 91,7 & 87,5 & 84,6 & 100 & 90 & 88,8 & 45,3 \\
\hline ASTURIAS & 0 & 0 & 1 & 0,5 & 2 & 1 & 4,5 & 56,25 & 92,1 & 100 & 96,9 & 92,3 & 100 & 80 & 93,8 & 65,9 \\
\hline BALEARES & 0 & 0 & 1 & 0,5 & 2 & 1,5 & 5 & 62,50 & 100 & 100 & 100 & 88,5 & 100 & 100 & 98,1 & 290 \\
\hline CANARIAS & 0,5 & 0 & 1 & 1 & 1 & 1 & 4,5 & 56,25 & 100 & 100 & 96,9 & 80,8 & 90 & 100 & 95 & 99,8 \\
\hline CANTABRIA & 0 & 0 & 1 & 0 & 1 & 0 & 2 & 25 & 100 & 83,3 & 100 & 84,6 & 100 & 100 & 95 & 51 \\
\hline CASTILLA LM & 0 & 0 & 1 & 0 & 1 & 0 & 2 & 25 & 89,5 & 100 & 90,6 & 84,6 & 100 & 100 & 90,6 & 19,2 \\
\hline CASTILLA LEÓN & 0 & 0 & 0,5 & 0,5 & 1 & 0 & 2 & 25 & 94,7 & 100 & 90,6 & 88,5 & 95 & 100 & 94,4 & 60,1 \\
\hline CATALUÑA & 0,5 & 0 & 0,5 & 0,5 & 2 & 1 & 4,5 & 56,25 & 100 & 100 & 96,9 & 92,3 & 100 & 100 & 98,1 & 12 \\
\hline COMU. VAL. & 0,5 & 0 & 1 & 1 & 2 & 1 & 5,5 & 68,75 & 100 & 100 & 84,4 & 84,6 & 100 & 100 & 94,4 & 44 \\
\hline EXTREMADURA & 0 & 0 & 0,5 & 0 & 1 & 0 & 1,5 & 18,75 & 89,5 & 91,7 & 87,5 & 84,6 & 85 & 90 & 88,1 & 54,6 \\
\hline GALICIA & 0,5 & 0 & 0,5 & 0,5 & 2 & 0 & 3,5 & 43,75 & 92,1 & 87,5 & 100 & 84,6 & 85 & 100 & 91,9 & 29,1 \\
\hline MADRID & 0 & 0 & 0,5 & 1 & 1 & 0,5 & 3 & 37,5 & 94,7 & 100 & 100 & 100 & 95 & 100 & 98,1 & 9,3 \\
\hline MURCIA & 0 & 0 & 0,5 & 0 & 1 & 1 & 2,5 & 31,25 & 100 & 95,8 & 100 & 92,3 & 95 & 95 & 96,9 & 13,6 \\
\hline NAVARRA & 0,5 & 0 & 1 & 1 & 2 & 1 & 5,5 & 68,75 & 86,8 & 95,8 & 75 & 80,8 & 80 & 70 & 81,9 & 0 \\
\hline PAIS VASCO & 0,5 & 2 & 1 & 0,5 & 1 & 1,5 & 6,5 & 81,25 & 100 & 100 & 100 & 100 & 100 & 100 & 100 & 9,1 \\
\hline LA RIOJA & 0 & 2 & 1 & 0 & 1 & 0 & 4 & 50 & 94,7 & 100 & 100 & 100 & 95 & 95 & 97,5 & 0 \\
\hline
\end{tabular}

Fuente: Vela y Zafra-Gómez (eds.) (2019), Transparencia Internacional España (2016), CGPJ (2018) y elaboración propia. 
Dada la heterogeneidad de los diferentes niveles de corrupción, auditoría y transparencia, resulta difícil establecer una relación clara entre las tres variables consideradas, por lo que, se presenta en la siguiente sección un análisis estadístico, a través del cual se pretende determinar la medida en que es posible corroborar esta cuestión.

\section{Análisis empírico: modelos y resultados}

En esta sección se analiza en qué medida el nivel de implantación y alcance de la auditoría externa y transparencia informativa en cada Comunidad se relaciona o no con los niveles de corrupción. Tras presentarse el estudio descriptivo de dichas variables y sus posibles correlaciones mediante un análisis exploratorio bivariante, se analizan los resultados de diferentes modelos de regresión lineal formulado con el propósito de explicar la variable corrupción.

Así, el análisis descriptivo de las variables nivel de implantación y alcance de la auditoria, nivel de transparencia y corrupción política se recoge en la Tabla 7 .

Tabla 7. Estadísticos descriptivos

\begin{tabular}{lrrr}
\hline \multicolumn{1}{c}{ Variable } & $\begin{array}{r}\text { Total GPA } \\
\text { S/100 }\end{array}$ & TP Global & $\begin{array}{c}\mathrm{N}^{\circ} \text { casos corrupción } \\
\text { ponderado }\end{array}$ \\
\hline Min & 18,75 & 81,90 & 0 \\
Q1 & 31,25 & 91,90 & 11,97 \\
Mediana & 50 & 95 & 43,96 \\
Media & 47,43 & 94,05 & 51,50 \\
Q3 & 56,25 & 97,50 & 60,13 \\
Max & 81,25 & 100 & 290 \\
Desv. Típica & 18,10 & 4,60 & 67,76 \\
Coef. Variación & $38,15 \%$ & $4,89 \%$ & $131,56 \%$ \\
Asimetría & 0,01 & $-1,04$ & 2,48 \\
Curtosis & $-1,19$ & 0,45 & 6,12 \\
\hline
\end{tabular}

El grado de planificación y alcance de la auditoría presenta una media del 47,43 sobre un valor máximo de 100. Es una variable centrada (asimetría prácticamente cero), con un coeficiente de variación (desviación típica dividida por la media) del 38\%, indicando variabilidad, pero no valores muy elevados. El coeficiente de curtosis negativo, pero mayor que -2 , nos indica una distribución ligeramente planicúrtica, es decir ligeramente plana. Por su parte, el indicador de transparencia global muestra una media elevada $(94,05$ sobre un valor máximo del indicador de 100), muy poca variabilidad (coeficiente de variación del 4,89\%), ligeramente asimétrica por la izquierda y apenas leptocúrtica, es decir ligeramente apuntada. Finalmente, el indicador de número de casos de corrupción escalado cada 10 millones de habitantes destaca por su elevada variabilidad, ya que el coeficiente de variación es superior al $100 \%$. Por otro lado, también es muy asimétrica por la derecha, indicando valores elevados, de hecho, el valor máximo de 290 supera la media más tres veces la desviación típica indicando la presencia de valores extremos por la derecha. Por último, presenta una distribución muy leptocúrtica, es decir muy apuntada.

Tal y como se ha explicado previamente, en primer lugar, se realiza un estudio exploratorio bivariante, para analizar las posibles relaciones entre alcance de auditoría, transparencia y corrupción. Así, se ha llevado a cabo un análisis exploratorio gráfico (Gráfico 1) que estudia la asociación entre el grado de planificación y alcance de la auditoría con la transparencia. De él se observa que la Comunidad Foral de Navarra presenta un punto influyente en dicha relación al presentar un valor extremadamente bajo en dicha relación.
Gráfico 1. Gráfico de dispersión Grado de planificación y alcance de la auditoria vs Transparencia global

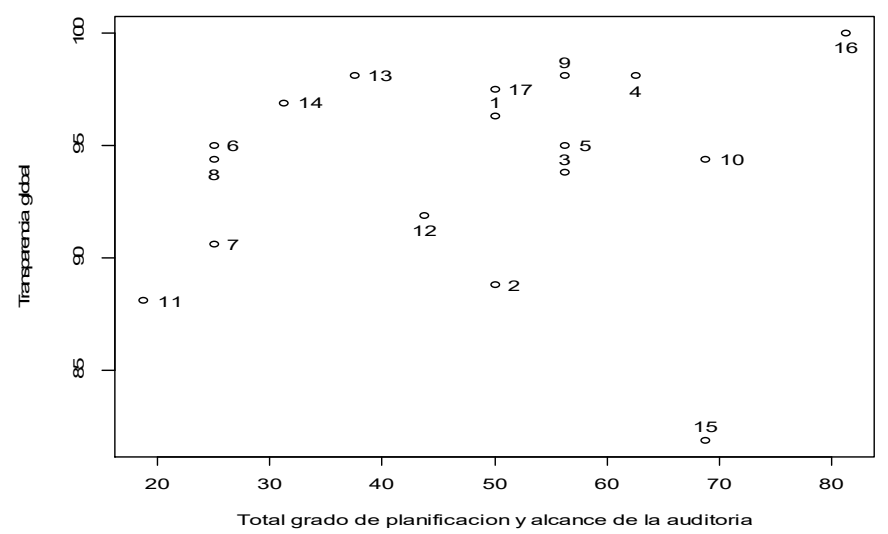

Leyenda: 1: Andalucía, 2: Aragón, 3: Asturias, 4: Baleares, 5: Canarias, 6: Cantabria, 7: Castilla LM, 8: Castilla León, 9: Cataluña, 10: Comunidad Valenciana, 11: Extremadura, 12: Galicia, 13: Madrid, 14: Murcia, 15: Navarra, 16: País Vasco, 17: La Rioja.

Por tanto, excluyendo a Navarra que es un caso singular en esta relación, hemos calculado el coeficiente de correlación lineal dando como resultado una correlación positiva del 50,46\% que resulta estadísticamente significativa (p valor del test de asociación entre muestras emparejadas de Pearson de 0,046).

De igual modo, hemos procedido a analizar la existencia o no de asociación entre auditoría y corrupción.

Gráfico 2. Gráfico de dispersión Grado de planificación y alcance de la auditoria vs corrupción.

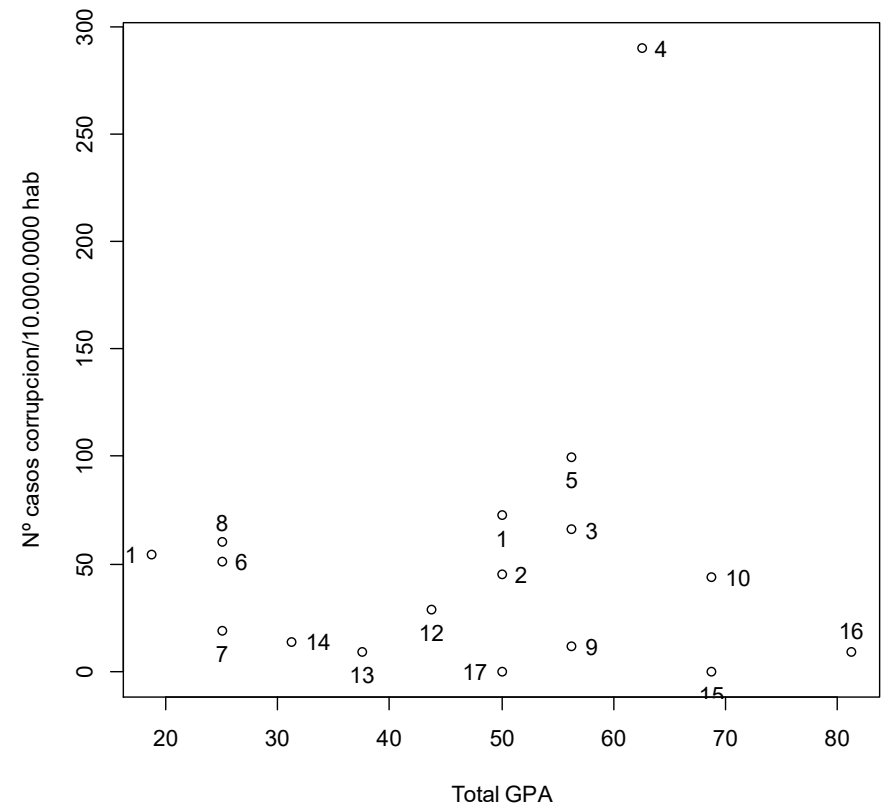

Leyenda: 1: Andalucía, 2: Aragón, 3: Asturias, 4: Baleares, 5: Canarias, 6: Cantabria, 7: Castilla LM, 8: Castilla León, 9: Cataluña, 10: Comunidad Valenciana, 11: Extremadura, 12: Galicia, 13: Madrid, 14: Murcia, 15: Navarra, 16: País Vasco, 17: La Rioja.

A partir del análisis gráfico, se ha descartado la comunidad de Baleares por ser un punto influyente en dicha relación al presentar un valor extremadamente alto. Aunque, no se puede observar gráficamente la existencia de asociación entre el nivel de auditoria y corrupción, se ha calculado el coeficiente de correlación lineal para las restantes 16 comunidades autónomas, resultando en una correlación negativa $(-15,34 \%)$ no significativa (p-valor: 0,5704). 
Asimismo, se ha llevado a cabo un estudio individual de la posible asociación de cada uno de los indicadores que componen el índice grado de planificación y alcance de la auditoría parcial y corrupción mediante un análisis de la varian$\mathrm{za}^{1}$. En relación con lo anterior, no se han detectado asociaciones estadísticamente significativas a excepción del indicador GIA2 que resulta prácticamente significativo al 10\% (pvalor $=0,101)$, donde las comunidades que aplican el Plan General de Contabilidad adaptado a PGCP 2010 presenta una media muy inferior de casos de corrupción ponderados $(4,57)$ frente a las comunidades que no han adaptado el Plan $(41,17)$.

Finalmente, respecto a la posible asociación entre transparencia y corrupción, después de un análisis gráfico (Gráfico 3), se han descartado las comunidades de Baleares y Navarra por ser puntos influyentes en dicha relación ${ }^{2}$. Excluyendo estos dos puntos se obtiene un coeficiente de correlación negativa $(-35,81 \%)$, que resulta no significativa (p-valor: 0,19$)$.

Gráfico 3. Gráfico de dispersión Transparencia global vs corrupción

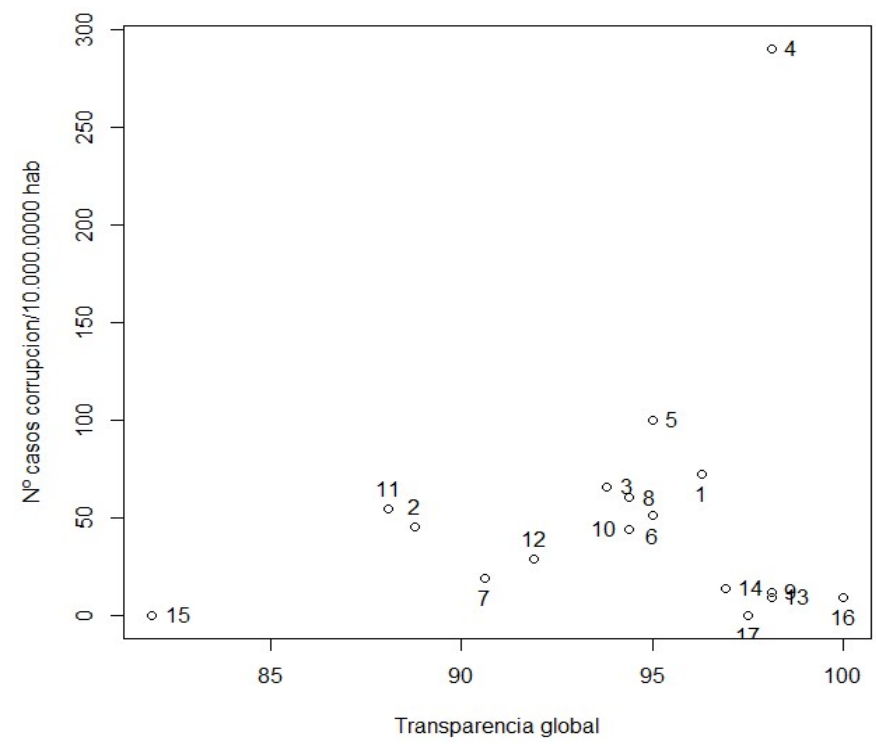

Leyenda: 1: Andalucía, 2: Aragón, 3: Asturias, 4: Baleares, 5: Canarias, 6: Cantabria, 7: Castilla LM, 8: Castilla León, 9: Cataluña, 10: Comunidad Valenciana, 11: Extremadura, 12: Galicia, 13: Madrid, 14: Murcia, 15: Navarra, 16: País Vasco, 17: La Rioja.

Por otro lado, se ha llevado a cabo un estudio de las correlaciones entre cada uno de los índices parciales de transparencia y el nivel de corrupción, resultando significativo uno de los que, a priori, se había considerado más relevantes para el objetivo del presente trabajo, en concreto, el índice de transparencia en convenios, subvenciones, y costes de los servicios. Dicho índice presenta una correlación negativa del 48,12\%, resultando significativa ( $\mathrm{p}$-valor $=0,069$ ).

Hasta el momento, se han analizado las relaciones entre auditoría, transparencia y corrupción de un modo bivariante, es decir, únicamente la posible relación entre dos variables. A continuación, se procederá a estudiar las relaciones conjun-

\footnotetext{
${ }^{1}$ Los distintos GIAs son variables no métricas y ordinales, por lo que hemos llevado a cabo un análisis de la varianza observando si los niveles de la variable siguen una relación positiva o negativa acorde al orden de los distintos niveles.

${ }^{2}$ Puesto que gráficamente la consideración de Baleares como punto influyente es obvia, pero no es tan obvio para el caso de Navarra, para evitar cualquier sesgo en la consideración de Navarra se ha llevado a cabo una regresión lineal de la variable transparencia sobre la variable corrupción como variable dependiente empleando el software R. Se han analizado los puntos influyentes con el comando de R "influence.measures" corroborando la consideración de Navarra como punto influyente.
}

tamente entre las tres variables, comenzando con un análisis gráfico, mediante un gráfico de dispersión tridimensional (Gráfico 4).

En el Gráfico 4 se puede observar cómo Baleares y Navarra están fuera de la nube de puntos. En el extremo inferior derecho de la nube de puntos se sitúa el País Vasco, que presenta elevados niveles de auditoria y transparencia, pero muy bajos niveles de corrupción.

Gráfico 4. Gráfico de dispersión tridimensional: auditoría, transparencia global, corrupción.

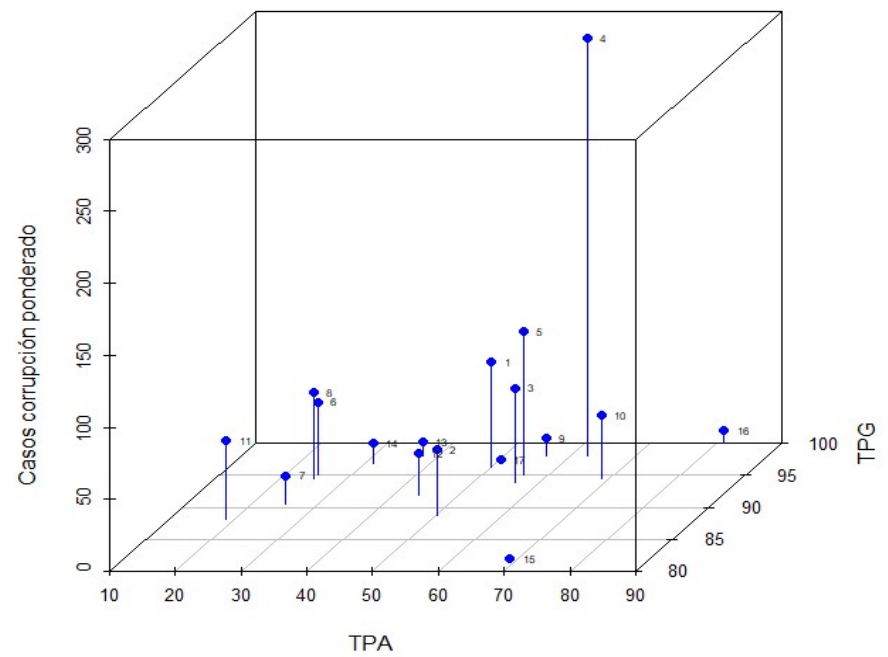

Leyenda: 1: Andalucía, 2: Aragón, 3: Asturias, 4: Baleares, 5: Canarias, 6: Cantabria, 7: Castilla LM, 8: Castilla León, 9: Cataluña, 10: Comunidad Valenciana, 11: Extremadura, 12: Galicia, 13: Madrid, 14: Murcia, 15: Navarra, 16: País Vasco, 17: La Extrem
Rioja.

Partiendo del análisis gráfico anterior, se ha estimado el siguiente modelo de regresión lineal para todas las comunidades autónomas excepto Baleares y Navarra por considerarse puntos influyentes ${ }^{3}$.

$$
C_{i}=\beta_{o}+\beta_{1} \mathrm{TPA}_{i}+\beta_{2} \mathrm{TPG}_{i}+\varepsilon_{i}
$$

Donde los casos de corrupción $\left(\mathrm{C}_{\mathrm{i}}\right)$ se explican por el Grado de planificación y alcance de la auditoria $\left(\mathrm{TPA}_{\mathrm{i}}\right)$ y por el índice de transparencia global $\left(\mathrm{TPG}_{\mathrm{i}}\right)$.

Tabla 8. Modelo de regresión $C_{i}=\beta_{o}+\beta_{1} T P A_{i}+\beta_{2} T P G_{i}+\varepsilon_{i}$

\begin{tabular}{lrrrr}
\hline & Coeficiente & Error estándar & T valor & $\operatorname{Pr}(>|\mathrm{t}|)$ \\
\hline Intercepto & 363.3156 & 226.7064 & 1.603 & 0.135 \\
TPA & 0.2441 & 0.4875 & 0.501 & 0.626 \\
TPG & -3.5443 & 2.4961 & -1.420 & 0.181 \\
\hline
\end{tabular}

Error estándar residual: 28.73 sobre 12 grados de libertad

$\mathrm{R}^{2}$ : $0.1461 \mathrm{R}^{2}$ ajustado: 0.003782

Estadístico F: 1.027 , p-valor: 0.3877

Los resultados se muestran en la Tabla 8 , donde se puede apreciar que ninguna de las variables del modelo resulta significativa ni tampoco el modelo. No obstante, es indicativo que el coeficiente del grado de planificación y alcance de la auditoria, aunque muy pequeño, resulta positivo, en contra de los resultados obtenidos por la literatura previa (Duflo et al., 2012, Duflo et al., 2013, Avis et al., 2018, Gustavson \& Sundström, 2018). Por su parte, aunque el coeficiente del

\footnotetext{
${ }^{3}$ Aunque el análisis gráfico es bastante esclarecedor, no obstante, hemos realizado el mismo modelo de regresión lineal para todas las Comunidades Autónomas analizado los puntos influyentes con el comando de $\mathrm{R}$ "influence.measures" corroborando la consideración de Baleares y Navarra como puntos influyentes.
} 
índice de transparencia global no resulta significativo, sí que presenta una relación negativa con el nivel de corrupción, tal y como se evidencia en estudios previos (Lindstedt \& Naurin, 2010, Montes \& Luna, 2020). La correlación entre auditoria y transparencia puede dar lugar a que se confundan los efectos, en todo caso el valor del coeficiente es muy cercano a cero y no resulta significativo.

Por otro lado, se ha buscado el mejor modelo, partiendo del modelo anterior (modelo completo mediante procedimiento backward) y desde el modelo nulo (procedimiento forward) coincidiendo en el modelo siguiente (Tabla 9), donde la única variable independiente es la transparencia global.

Tabla 9. Modelo de regresión $C_{i}=\beta_{o}+\beta_{1} T P G_{i}+\varepsilon_{i}$

\begin{tabular}{lrrrr}
\hline & Coeficiente & Error estándar & $\mathrm{T}$ valor & $\operatorname{Pr}(>|\mathrm{t}|)$ \\
\hline Intercepto & 318.486 & 202.194 & -1.490 & 0.16 \\
TPG & -2.924 & 0.1451 & -1.383 & 0.19 \\
\hline
\end{tabular}

Error estándar residual: 27.89 sobre 13 grados de libertad

$\mathrm{R}^{2}$ : $0.1283 \mathrm{R}^{2}$ ajustado: 0.0612

Estadístico F: 1.913, p-valor: 0.19

Aunque el modelo sigue sin resultar significativo, sus resultados son más satisfactorios y el $\mathrm{R}^{2}$ ajustado aumenta. No obstante, el bajo valor es indicativo de las múltiples variables que pueden explicar la corrupción.

Además, teniendo en cuenta el estudio de correlaciones anterior, se ha considerado si los indicadores parciales de auditoria y de transparencia pudieran explicar mejor la corrupción que los indicadores globales. En este sentido, se propone el siguiente modelo:

$$
C_{i}=\beta_{o}+\beta_{1} \mathrm{GIA}_{i}+\beta_{2} \mathrm{TPD}_{i}+\varepsilon_{i}
$$

Donde los casos de corrupción $\left(\mathrm{C}_{\mathrm{i}}\right)$ se explican por el indicador GIA2 Aplicación del Plan General de Contabilidad adaptado a PGCP2010 y el índice de transparencia en convenios, subvenciones, y costes de los servicios (TPD), siendo la variable GIA2 un factor con dos niveles y el índice TPD una variable continua.

Tabla 10. Modelo de regresión $C_{i}=\beta_{o}+\beta_{1} G I A 2_{i}+\beta_{2} T P D_{i}+\varepsilon_{i}$

\begin{tabular}{lrrrr}
\hline & Coeficiente & Error estándar & $\mathrm{T}$ valor & $\operatorname{Pr}(>|\mathrm{t}|)$ \\
\hline Intercepto & 155.502 & 108.313 & 1.436 & 0.177 \\
GIA2 & -25.687 & 24.060 & -1.068 & 0.307 \\
TPD & -1.252 & 1.218 & -1.029 & 0.324 \\
\hline
\end{tabular}

Error estándar residual: 26.05 sobre 12 grados de libertad

$\mathrm{R}^{2}$ : $0.2982 \mathrm{R}^{2}$ ajustado: 0.1813

Estadístico F: 2.55, p-valor: 0.1194

Los coeficientes de dichas variables presentan signos negativos, aunque ninguna de las variables resulta significativa y por tanto tampoco el modelo resulta significativo, aunque está más cerca de considerarse significativo que los anteriores y presenta un mejor $\mathrm{R}^{2}$ ajustado.

Del mismo modo en que se ha procedido anteriormente, tomando como base este último modelo, se busca el mejor modelo mediante un procedimiento backward y desde el modelo nulo (procedimiento forward) coincidiendo en el modelo siguiente (Tabla 11), donde la única variable independiente es la Aplicación del Plan General de Contabilidad adaptado a PGCP2010.

En este caso, el modelo resulta significativo con respecto a la variable aplicación del Plan General de Contabilidad adaptado a PGCP 2010, que presenta un coeficiente negativo de 39,77 a un nivel de significatividad del $10 \%$, indicando que al pasar de una Comunidad Autónoma que no ha adaptado
Tabla 11. Modelo de regresión $C_{i}=\beta_{o}+\beta_{1} G I A 2_{i}+\varepsilon_{i}$

\begin{tabular}{lrrrr}
\hline & Coeficiente & Error estándar & T valor & $\operatorname{Pr}(>|\mathrm{t}|)$ \\
\hline Intercepto & 44.34 & 7.24 & 6.125 & $3.63 \mathrm{e}-05 * * *$ \\
GIA2 & -39.77 & 19.83 & -2.006 & 0.0661 \\
\hline
\end{tabular}

Error estándar residual: 26.1 sobre 13 grados de libertad

$\mathrm{R}^{2}: 0.2364 \mathrm{R}^{2}$ ajustado: 0.1776

Estadístico F: 4.024, p-valor: 0.06613

Significativa al: ‘***' $0.1 \%$; ‘**' $1 \%$; ‘*' $5 \%$; ‘' $10 \%$

el Plan a una que sí lo ha hecho, los casos de corrupción descienden en término medio en 39,77 casos cada 10.000.000 habitantes. En este sentido, cabe cierta discusión respecto a este resultado que debe interpretarse con prudencia, teniendo en cuenta que de esta relación cabría presumir que dicha adaptación representa sin duda una clara voluntad de disciplina y de avanzar en calidad y rigor en el ámbito normativo, especialmente en lo que se refiere a su cumplimiento. Lo que podría interpretarse, en línea con los resultados de Gustavson \& Sundström (2018), con que el nivel de calidad de auditoría contribuye a la reducción de los casos de corrupción política.

Finalmente, se han comprobado los supuestos de normalidad y homocedasticidad. En relación con lo anterior, el test de Shapiro y el test de Breusch-Pagan no rechazan respectivamente las hipótesis nulas de normalidad y homocedasticidad.

\section{Conclusiones}

El control y la supervisión en las Administraciones Públicas cada vez es más necesario debido a las mayores exigencias ciudadanas, provocadas en gran medida por circunstancias sobrevenidas como la crisis económica y financiera del año 2008. Por ello, en este estudio se ha llevado a cabo un estudio exploratorio de la relación entre la corrupción en las administraciones públicas, el nivel de auditoría externa y el grado de transparencia en las Comunidades Autónomas.

Para ello, se ha realizado en primer lugar, un estudio bivariante de la correlación de estas variables, proponiéndose posteriormente, dos modelos multivariantes. En el primero de ellos, se considera el nivel de auditoría y la transparencia como variables explicativas de la corrupción; mientras que, en el segundo se incluyen dos variables parciales del nivel de auditoría y transparencia, que son la aplicación del Plan General de Contabilidad Adaptado al PGCP 2010 y el índice de transparencia en convenios, subvenciones, y costes de los servicios, respectivamente.

Así, los resultados del análisis de correlaciones muestran que la auditoría está directamente relacionada con la transparencia, mientras que las asociaciones entre auditoría y corrupción y transparencia y corrupción no resultan significativas. Estos resultados sugieren que la relación entre auditoría, transparencia y corrupción es compleja y presumiblemente dependiente de factores individuales de cada Comunidad Autónoma, que van desde Comunidades Autónomas con altos niveles de auditoría y transparencia con muy bajos niveles de corrupción (País Vasco) a Comunidades Autónomas con altos niveles de auditoría, bajos niveles de transparencia y bajos niveles de corrupción (Navarra).

Finalmente, respecto al objetivo principal del presente trabajo, el análisis del modelo multivariante no ha evidenciado un efecto significativo de la transparencia ni de la auditoría sobre la corrupción. Sin embargo, de los modelos alternativos propuestos sobre indicadores parciales, se obtiene un modelo significativo cuando la única variable independiente es el factor aplicación del Plan General de Contabilidad Adaptado al PGCP 2010. No obstante, las conclusiones han de for- 
mularse con cautela. Desde la lógica, la aplicación o no del PGC adaptado al PGCP 2010 puede no ser un factor que influya directamente de forma negativa en la corrupción, aunque puede entenderse como un factor indicativo del conjunto de características individuales de la Comunidad Autónoma que influyen negativamente en la corrupción, como una administración más ágil, más respetuosa con las normas, etc. Y es que, en la realidad, la mayoría de casos de corrupción que llegan a los tribunales son el resultado de denuncias llevadas a cabo por el Ministerio Fiscal, particulares o partidos políticos que en general no emanan de salvedades o recomendaciones que los informes de auditoría desarrollan.

En este sentido, es evidente que los informes de auditoría en el sector público no se diseñan ni estructuran con el objetivo de detectar fraudes y casos de corrupción. Su finalidad, en el caso de las auditorías de legalidad y regularidad se orienta claramente hacia la rendición de cuentas y el objetivo de imagen fiel. Por lo que, los resultados obtenidos en el presente trabajo invitan a reflexionar sobre su utilidad y la medida en que los mismos son o no utilizados por sus potenciales usuarios, en función de sus necesidades de información, lo que además podría ser abordado con más detalle en futuros trabajos de investigación.

Asimismo, las diferencias existentes en relación con los plazos de aprobación de los informes de la Cuenta General de cada Comunidad Autónoma encierran una gran trascendencia para que la información suministrada tanto en las Cuentas como en los informes de Auditoría resulte verdaderamente útil, relevante y comparable. Resulta sin duda deseable, y cada vez más inaplazable, abordar la homogeneización de dichas fechas de aprobación. A este respecto, sería oportuno que bien el Consejo de Política Fiscal y Financiera o el propio Ministerio de Hacienda analizaran y remediaran esta cuestión en beneficio del conjunto de los usuarios de la información contable. A este respecto, no debe olvidarse que la información que se utiliza a nivel macroeconómico para cuantificar tanto el déficit como el endeudamiento de las diferentes Comunidades emana de la contenida en sus propias Cuentas Generales.

Por tanto, la reducción de los niveles de heterogeneidad existentes con respecto al grado de desarrollo de la auditoría externa resulta ciertamente deseable a nivel autonómico, con independencia del grado de transparencia o de corrupción. En este sentido, la necesidad de una mayor armonización en el ámbito de la auditoría de las Comunidades Autónomas españolas constituye un aspecto que debería abordarse en el marco del Consejo de Política Fiscal y Financiera, especialmente teniendo en cuenta una futura reforma del sistema de financiación autonómica que en la actualidad resulta absolutamente tan inaplazable como necesaria, para reducir la brecha existente en los recursos per cápita que cada Comunidad recibe en la actualidad.

Como colación, es necesario aclarar que con unos niveles de implantación de la auditoría externa más homogéneos, el debate en torno al endeudamiento, el déficit y las necesidades de financiación de cada Comunidad podría sin duda enriquecerse, permitiendo tanto a las agencias de calificación como al sistema financiero y resto de usuarios contar con una herramienta de análisis que hasta ahora, al menos, parece no haber sido utilizada con la suficiente extensión dada la heterogeneidad señalada en su alcance, ya que como usuarios de la información contable, su labor resultaría ciertamente beneficiada.

Finalmente, es necesario remarcar diversas limitaciones que presenta el estudio, y es que la población de estudio es reducida al centrarse exclusivamente en las Comunidades Autó- nomas y los datos relativos a los casos de corrupción política incluyen tanto los acontecidos a nivel autonómico como local. Se trata, por tanto, de un primer estudio de carácter preliminar, por lo que futuros trabajos de investigación requieren profundizar en el análisis empírico de esta cuestión, alargando el horizonte temporal y considerando además el efecto que otras variables pueden tener sobre los niveles de corrupción autonómica. Asimismo, nos parece adecuado destacar, con respecto a posteriores ejercicios económicos, la medida en que un mayor o menor grado de implantación de la auditoría a nivel autonómico puede influir o no en aspectos como la situación financiera de las diferentes regiones o las calificaciones obtenidas en los diferentes informes de "rating" que las diferentes agencias de calificación puedan llevar a cabo.

\section{Financiación}

Esta investigación no recibió ninguna subvención específica de organismos de financiación de los sectores público, comercial o sin ánimo de lucro.

\section{Conflicto de intereses}

Los autores declaran no tener ningún conflicto de intereses.

\section{Referencias}

Alonso, M. L., \& Garcia-Garcia, J. (2014). Evaluación de la Transparencia Municipal en el Principado de Asturias. Auditoría Pública, 64, 75-86.

Andersen, T. B. (2009). E-government as an anti-corruption strategy. Information Economics and Policy, 21(3), 201210. https://doi.org/10.1016/j.infoecopol.2008.11.003

Avis, E., Ferraz, C., \& Finan, F (2018). Do Government Audits Reduce Corruption? Estimating the Impacts of Exposing Corrupt Politicians. Journal of Political Economy, 126(5), 1912-1964. https://doi.org/10.1086/699209

Bellod, J. (2008). Significado y evaluación de la transparencia presupuestaria en las Comunidades Autónomas. Auditoría Pública, 45, 103-115.

Benito, B., Guillamón, M. D., Ríos, A. M., \& Bastida, F. (2018). Can salaries and re-election prevent political corruption? An empirical evidence. Revista de Contabilidad-Spanish Accounting Review, 21(1), 19-27. https://doi.org/10.1016/ j.rcsar.2017.04.003

Benito, B., Guillamón, M. D., \& Ríos, A. M. (2021). Transparency and efficient management in local governments. $\mathrm{Ci}$ ties, 115, 103234. https://doi.org/10.1016/j.cities.2021. 103234

Campos, J. E., \& Pradhan, S. (2007). The many faces of corruption: tracking vulnerabilities at the sector level. Washington, DC: World Bank. Recuperado de https://openknowledge. worldbank.org/bitstream/handle/10986/6848/ 399850REPLACEM101OFFICIALOUSE0ONLY1.pdf? sequence $=1$ \&isAllowed $=\mathrm{y}$

Chaikin, D., \& Sharman, J. (2009). Corruption and money laundering: a symbiotic relationship. New York, USA: Palgrave Macmillan US.

Consejo General del Poder Judicial (CGPJ) (2018): Repositorio de datos sobre procesos por corrupción. Poder judicial. Recuperado el 23/05/2019 de https: //www.poderjudicial.es/cgpj/es/Temas/Transparencia/ 
ch.Repositorio-de-datos-sobre-procesos-por-corrupcion. formato1/

Cuadrado-Ballesteros, B., \& Peña-Miguel, N. (2020). Corruption perception following privatization reforms: The moderating role of the quality of governance. Revista de Contabilidad-Spanish Accounting Review, 23(1), 127-137. https://doi.org/10.6018/rcsar.361041

Cuillier, D., \& Piotrowski, S. J. (2009). Internet informationseeking and its relation to support for access to government records. Government Information Quarterly, 26(3), 441-449. 441-449. https://doi.org/10.1016/j.giq.2009. 03.001

Curry, D. S. D., Jilke, S., Hammerschmid, G., \& Van de Walle, S. (2014). Coordinating for Cohesion in the Public Sector of the Future: Final summary policy brief.

Da Cruz, N. F., \& Marques, R. (2014). Scorecards for Sustainable Local Governments."Cities: The International Journal of Urban Policy and Planning, 39, 165-170. https: //doi.org/10.1016/j.cities.2014.01.001.

Da Cruz, N. F., Tavares, A. F., Marques, R. C., Jorge, S., \& de Sousa, L. (2016). Measuring local government transparency. Public Management Review, 18(6), 866-893. https: //doi.org/10.1080/14719037.2015.1051572

Dasí, R.M., Gimeno, A., \& Vela, J.M. (2018). The Recent Reform of Spanish Local Governmental Accounting: A Critical Perspective from Local Governmental Accountants as Internal Users of Budgeting and Financial Accounting Information. Lex Localis. Journal of Local SelfGovernment, 16(3), 453-473. https://doi.org/10.4335/ 16.3.453-476(2018)

De Fine Licht, J. (2011). Do we really want to know? The potentially negative effect of transparency in decision making on perceived legitimacy. Scandinavian Political Studies, 34(3), 183-201. https://doi.org/10.1111/j. 1467-9477.2011.00268.x

De Herrera, M. A. C. (2010). Sobre corrupción y transparencia. Auditoría Pública, (50), 11-14. Por tanto, en el texto se debe cambiar la cita por: De Herrera, 2010.

Della Porta, D. (2017). Corrupt exchanges: Actors, resources, and mechanisms of political corruption. London, UK: Routledge.

Duflo, E., Greenstone, M., Pande, R., \& Ryan, N. (2013). Truth-telling by third-party auditors and the response of polluting firms: Experimental evidence from India. Quarterly Journal of Economics, 128, 1499-1545. https://doi. org/10.1093/qje/qjt024.

Duflo, E., Hanna, R., \& Ryan, S. P. (2012). Incentives work: Getting teachers to come to school. American Economic Review, 102, 1241-1278. https://doi.org/10.1257/aer.102. 4.1241

Garrido-Rodríguez, J. C., López-Hernández, A. M., \& ZafraGómez, J. L. (2019). The impact of explanatory factors on a bidimensional model of transparency in Spanish local government. Government Information Quarterly, 36(1), 154-165. https://doi.org/10.1016/j.giq.2018.10.010

Garrido-Rodríguez, J. C., Zafra-Gomez, J. L., \& LópezHernández, A. (2017). Measuring Local Government Transparency. Influence of Political Sign in Multidimensional Analysis. Lex Localis-Journal of Local SelfGovernment, 15(4), 889-917. https://doi.org/10.4335/ 15.4.889-917(2017)

Golden, M. A., \& Picci, L. (2005). Proposal for a new measure of corruption, illustrated with Italian data. Economics \& Politics, 17, 37-75. https://doi.org/10.1111/j.1468-0343. 2005.00146.X

Grimmelikhuijsen, S., Porumbescu, G., Hong, B., \& Im, T.
(2013). The effect of transparency on trust in government: A crossnational comparative experiment. Public Administration Review, 73(4), 575-586. https://doi.org/10.1111/ puar. 12047

Guillamón, M. D., Bastida, F., \& Benito, B. (2011). The determinants of local government's financial transparency. Local Government Studies, 37(4), 391-406. https://doi.org/ $10.1080 / 03003930.2011 .588704$

Gustavson, M., \& Sundström, A. (2018). Organizing the Audit Society: Does Good Auditing Generate Less Public Sector Corruption? Administration \& Society, 50(10), 15081532. https://doi.org/10.1177/0095399716674306

Heidenheimer, A., Johnston, M., \& Le Vine, V. (eds.) (1989). Political corruption: a handbook. New Brunswick, NJ: Transaction Publishers.

Johnston, M., (1996). The search for definitions: the vitality of politics and the issue of corruption. International Social Science Journal, 149 (3), 321-335. https://doi.org/ 10.1111/1468-2451.00035

Johnston, M. (2017). Political corruption: readings in comparative analysis. London, UK: Routledge.

Kaufmann, D. (2000). Corrupción y reforma institucional: el poder de la evidencia empírica. Revista Perspectivas, 3(2), 367-387.

Kaufmann, D., \& Kraay, A. (2002). Growth without Governance", Policy Research Working Paper n. 3077, Washington, USA: World Bank.

Kolstad, I., \& Wiig, A. (2009). Is transparency the key to reducing corruption in resource-rich countries? World development, 37(3), 521-532. https://doi.org/10.1016/j. worlddev.2008.07.002

Lambsdorff, J. G. (2005). Consequences and causes of corruption: What do we know from a cross- section of countries? Passauer Diskussionspapiere: Volkswirtschaftliche Reihe. Recuperado de https://www.econstor. eu/bitstream/10419/55031/1/684238772.pdf

Lindstedt, C., \& Naurin, D. (2010). Transparency is not enough: Making transparency effective in reducing corruption. International Political Science Review, 31, 301322. https://doi.org/10.1177/0192512110377602

Lizcano, J. (2016). Transparencia de ayuntamientos y diputaciones: la visión de transparencia internacional. En X. Forcadell Esteller \& M. Villoria Mendieta (Eds.), Buen Gobierno Transparencia e Integridad Institucional en el Gobierno Local (pp. 126-152). Barcelona: Tecnos.

López-Hernández, A. M., Zafra-Gómez, J. L., Plata-Díaz, A. M., \& de la Higuera-Molina, E. J. (2018). Modeling fiscal stress and contracting out in local government: The influence of time, financial condition, and the great recession. The American Review of Public Administration, 48(6), 565-583. https://doi.org/10.1177/0275074017699276

Magdaleno, M. L. A., \& García-García, J. (2014). Evaluación de la transparencia municipal en el Principado de Asturias. Auditoría pública, 64, 75-86.

Matheson, A. (2002). Better Public sector governance: the rationale for budgeting and accounting reform in western nations, in models of public budgeting and accounting reform. OECD Journal on budgeting, 2(1), 37-49.

Meijer, A. (2003). Transparent Government: Parliamentary and Legal Accountability in an Information Age. Information Polity, 8(1-2), 67-78.

Montes, G. C., \& Luna, P. H. (2020). Fiscal transparency, legal system and perception of the control on corruption: empirical evidence from panel data. Empirical Economics, 60, 2005-2037. https://doi.org/10.1007/ 


\section{s00181-020-01849-9}

Ortiz de Zárate, A. (2012). Tres tipos de Transparencia, Administración en red. https://eadminblog.net/2012/03/28/ tres-tipos-de-transparencia

Ormaetxea, I. G. (2014). Comentarios a la nueva regulación de la transparencia pública en España. Administración de Andalucía: revista andaluza de administración pública, 89, 379-412.

Pérez-López, G. (2019). El nivel de auditoría y fiscalización implementado en la Comunidad Autónoma de Castilla la Mancha. En La Investigación sobre la Auditoría en el Sector Público en España (pp. 159-168). Madrid: Instituto de Censores Jurados de Cuentas de España.

Pilcher, R., Taplin, R., \& Joseph, C. (2008). Sustainability reporting on local authority websites within an institutional theory framework. En R. Burritt (Ed), The 7th Australiasian conference for social and environmental accounting research (pp. 510-531). Adelaide, Australia: Center for Accounting Governance and Sustainability.

Reylea, H. C. (2009). Federal freedom of information policy: Highlights of recent developments. Government Information Quarterly, 26, 314-320. https://doi.org/10.1016/j. giq.2008.12.001

Ríos, A. M; Benito, B., \& Bastida, F. (2017). Factors Explaining Public Participation in the Central Government Budget Process. Australian Journal of Public Administration, 76(1), 48-64. https://doi.org/10.1111/1467-8500. 12197

Ríos, A.M., Redondo-López, A.M. \& Benito, B. (2019). La transparencia presupuestaria en las comunidades autónomas españolas. CIRIEC-España, Revista de Economía Pública, Social y Cooperativa, 96, p.281-310.

Royo, S., Yetano, A., \& García-Lacalle, J. (2019). Estilos de rendición de cuentas en empresas públicas: el bueno, el malo, el feo ... y la guapa. Revista de Contabilidad - Spanish Accounting Review, 22(2), 156-170. https://doi.org/ 10.6018/rcsar.382231

Shim, D. C., \& Eom, T. H. (2008). E-government and anticorruption: Empirical analysis of international data. International Journal of Public Administration, 31(3), 298-316. https://doi.org/10.1080/01900690701590553

Tanzi, V. (1998). Corruption around the world: Causes, consequences, scope, and cures. Staff Papers, 45(4), 559-594. https://doi.org/10.2307/3867585

Transparency International España (2016a). Índices de Transparencia de las Comunidades Autónomas INCAU. Metodología Utilizada. https://transparencia.org.es/incau-2016/

Transparency International España (2016b). Índices de Transparencia España. https://transparencia.org.es/ indices-transparencia-espana/

Transparency International. (2015). Local Governance Integrity: Principles and Standards. Berlin: Transparency International.

Transparency International (2018). What is corruption? https://www.transparency.org/what-is-corruption

Treisman, D. (2007). What Have We Learned About the Causes of Corruption from Ten Years of Cross-National Empirical Research? Annual Review of Political Science, 10, 211-244. https://doi.org/10.1146/annurev.polisci. 10.081205 .095418

Van de Walle, S., \& Hammerschmid, G. (2011). Coordinating for cohesion in the public sector of the future. COCOPS Project Background Paper.

Van Klaveren, J. (1989). The concept of corruption. En A. J. Heidenheimer, M. Johnston \& V. T. LeVine (Eds.) Political
Corruption: A Handbook. New Brunswick, N.J.: Transaction Books.

Villoria, M. (2015). El largo camino hacia la transparencia en los Ayuntamientos españoles. El consultor de los ayuntamientos, 18, 1983-2001.

Vela, J.M., \& Zafra-Gómez, J.L. (2019). La Investigación sobre la Auditoría en el Sector Público en España. Madrid: Instituto de Censores Jurados de Cuentas de España.

Vela, J.M., Polo, F., \& Pérez M.S. (2016). La reforma de la Contabilidad de la Administración Local española: algunas reflexiones críticas en torno a las nuevas instrucciones de contabilidad. CIRIEC-España, Revista de economía pública, social y cooperativa, 86, 221-250.

Villoria, M., \& Cruz-Rubio, C.N. (2016). Gobierno abierto, transparencia y rendición de cuentas: marco conceptual. En M. Villoria Mendieta, X. Forcadell Esteller \& L. Baena García (Eds.), Buen Gobierno, Transparencia e Integridad Institucional en el Gobierno Local (pp. 80-103). Barcelona: Tecnos.

Viñas, J. (2018). La transparencia y el control en el sector público: £hacia un cambio en la auditoría pública local? Auditoría Pública, 71, 33-40.

Zafra-Gómez, J.L. (2019). El nivel de auditoría y fiscalización implementado en la Comunidad Autónoma de Andalucía. En Vela J.M. \& Zafra-Gómez J.L. (Eds.), La Investigación sobre la Auditoría en el Sector Público en España (pp. 7784). Madrid: Instituto de Censores Jurados de Cuentas de España. 